\title{
Decarboxylative Anti-Michael Addition to Olefins Mediated by Photoredox Catalysis
}

\author{
Gabrielle H. Lovett and Brian A. Sparling \\ Department of Medicinal Chemistry, Amgen Inc. \\ Cambridge, MA, 02142 USA
}

\section{Table of Contents}

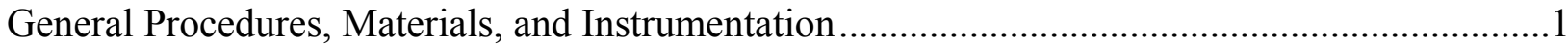

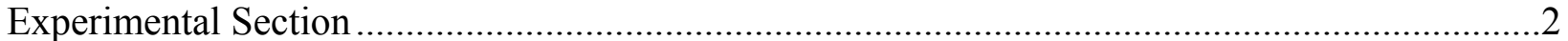

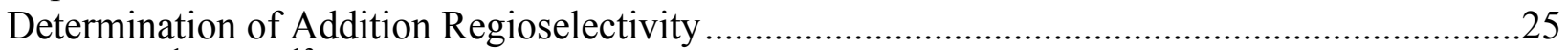

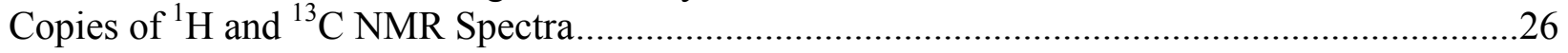

General Procedures, Materials, and Instrumentation. Commercial reagents and solvents were used as received. NMR spectra were recorded with Bruker AV III $500 \mathrm{MHz}$ and Bruker Avance $1400 \mathrm{MHz}$ spectrometers, are reported in parts per million $(\delta)$, and are calibrated using residual non-deuterated solvent as an internal reference: $\mathrm{CDCl}_{3}, \delta 7.26\left(\mathrm{CHCl}_{3}\right) ; d_{6}-\mathrm{DMSO}, \delta$ $2.50\left(d_{5}\right.$-DMSO); $\mathrm{CD}_{3} \mathrm{CN}, \delta 1.94\left(d_{2}-\mathrm{MeCN}\right)$. Data for ${ }^{1} \mathrm{H}$ NMR spectra are reported as follows: chemical shift (multiplicity, coupling constants, integration). Multiplicities are reported as follows: $\mathrm{s}=$ singlet; $\mathrm{d}=$ doublet; $\mathrm{t}=$ triplet; $\mathrm{q}=$ quartet; $\mathrm{m}=$ multiplet; $\mathrm{br}=$ broad, or combinations thereof. Data for ${ }^{13} \mathrm{C}$ NMR spectra are reported in parts per million $(\delta)$ and are referenced from the central peak of the carbon resonance of the solvent: $\mathrm{CDCl}_{3}, \delta 77.00 ; d_{6^{-}}$ DMSO, $\delta 39.52 ; \mathrm{CD}_{3} \mathrm{CN}, \delta 118.69\left(\mathrm{CD}_{3} \underline{\mathrm{CN}}\right)$. Infrared (IR) data were recorded on a Thermo Nicolet Avatar 360 FT-IR equipped with a Nicolet Smart Golden Gate, Diamond Crystal with a ZnSe focusing element. High-resolution mass spectra (HRMS) were recorded using electrospray ionization (ESI) mass spectroscopy on an Agilent 1100 Series HPLC system. Normal-phase chromatography was performed on a Biotage Isolera One system. Reverse phase chromatography was performed on a Waters Gilson preparatory HPLC system. Blue LED light was generated using two Kessil H150 LED Grow Lights flanking a stir plate, leaving approximately 2-4 inches of space between the lights and the reaction vials (Figure S1). 

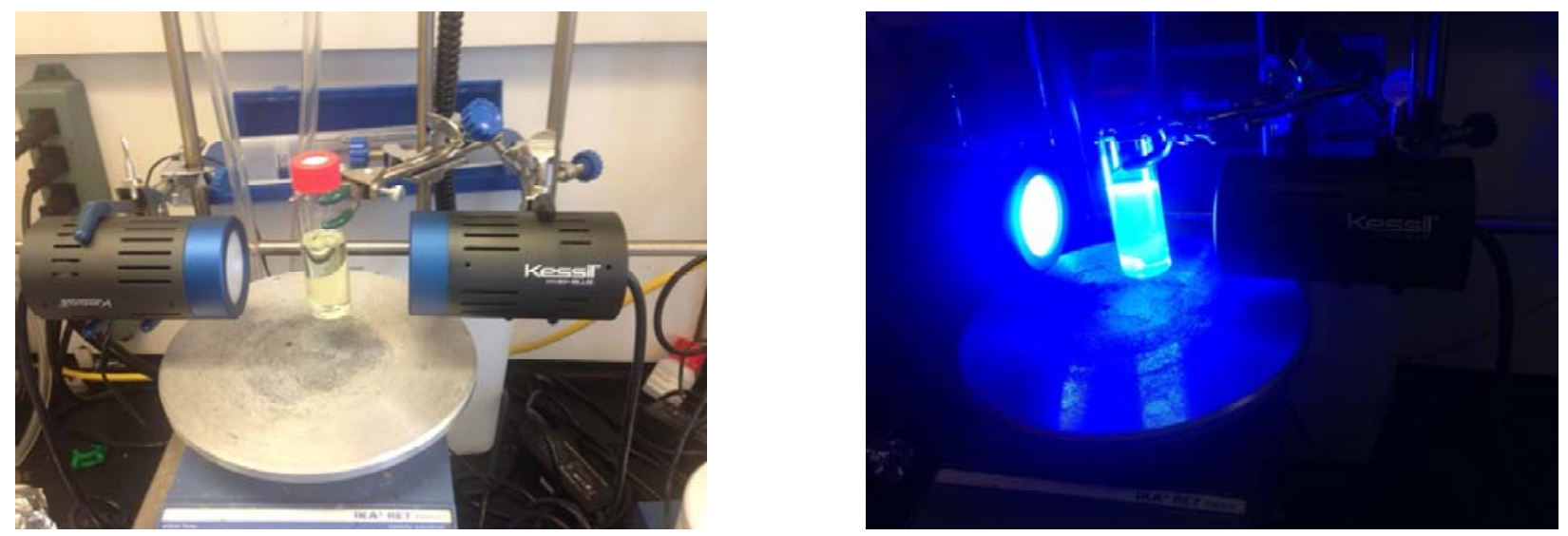

Figure S1. Photoredox reaction set up.

\section{Experimental Section.}

\section{General procedure for the decarboxylative anti-Michael olefin addition reaction:}

A DMSO $(15 \mathrm{~mL}, 0.02 \mathrm{M})$ solution of a radical acceptor $(0.314 \mathrm{mmol}, 1$ equiv), a carboxylic acid (0.471 mmol, 1.5 equiv), cesium carbonate (154 $\mathrm{mg}, 0.471 \mathrm{mmol}, 1.5$ equiv), and $\left[\operatorname{Ir}\left(\mathrm{dFCF}_{3} \text { ppy }\right)_{2}(\mathrm{dtbbpy})\right] \mathrm{PF}_{6}(\mathbf{1}, 3.5 \mathrm{mg}, 3.1 \mu \mathrm{mol}, 0.01$ equiv $)$ in a $40-\mathrm{mL}$ scintillation vial was sparged for 15 min with $\mathrm{N}_{2}$ and then irradiated with blue LED light while stirring. The irradiation caused the temperature of the reaction to raise to $45{ }^{\circ} \mathrm{C}$. After a specified period of time, the reaction was diluted with water and extracted four times with EtOAc. The organic extracts were combined, washed twice with water, dried over $\mathrm{Na}_{2} \mathrm{SO}_{4}$, filtered, and concentrated in vacuo. Column chromatography (10 g Biotage Snap Ultra column, 0-100\% EtOAc/heptane) afforded the product. 


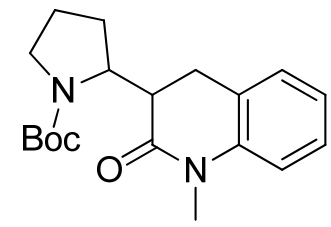

$( \pm)-4$

( \pm )-tert-Butyl 2-(1-methyl-2-oxo-1,2,3,4-tetrahydroquinolin-3-yl)pyrrolidine-1-carboxylate (4):

According to the general procedure, 1-methyl-2-quinolinone (3, $0.050 \mathrm{~g}, 0.314 \mathrm{mmol}), \mathrm{N}$-(tertbutoxycarbonyl)-L-proline $(2,0.101 \mathrm{~g}, 0.471 \mathrm{mmol})$, cesium carbonate $(154 \mathrm{mg}, 0.471 \mathrm{mmol})$, and $\left[\operatorname{Ir}\left(\mathrm{dFCF}_{3} \text { ppy }\right)_{2}(\mathrm{dtbbpy})\right] \mathrm{PF}_{6}(\mathbf{1}, 3.5 \mathrm{mg}, 3.1 \mu \mathrm{mol})$ in DMSO $(15 \mathrm{~mL})$ were used, and the reaction was stirred for $6 \mathrm{~h}$. Column chromatography afforded $92.6 \mathrm{mg}$ of $4(0.280 \mathrm{mmol}, 89 \%$ yield) as a white amorphous solid.

${ }^{1}$ H NMR (500 MHz, d -DMSO) $^{-}$: 7.25 (d, J=6.7 Hz, 2H), 7.07 (br s, 1H), 7.00 (d, J=6.2 Hz, $1 \mathrm{H}), 4.33-3.97(\mathrm{~m}, 1 \mathrm{H}), 3.46-3.10(\mathrm{~m}, 5 \mathrm{H}), 2.10-1.66(\mathrm{~m}, 4 \mathrm{H}), 1.47-1.17(\mathrm{~m}, 10 \mathrm{H})$ (mixture of diastereomers and rotamers).

${ }^{13}$ C NMR (126 MHz, $d_{6}$-DMSO) $\delta: 173.61,169.94,153.59,139.89,127.78,127.38,125.47$, $125.09,124.99,122.61,122.43,122.35,114.69,114.56,114.40,81.37,79.93,78.48,78.37$, $57.03,56.09,55.84,46.65,46.26,46.14,43.23,43.05,42.33,32.53,29.29,29.19,29.13,28.12$, $27.67,26.89,25.30,25.03,23.63,23.22,22.93,22.11,16.92$ (mixture of diastereomers and rotamers).

FTIR (thin film) $v_{\max }: 2972,2929,2882,1685,1668,1603,1472,1389,1364,1263,1160,1121$, $753 \mathrm{~cm}^{-1}$.

HRMS-ESI (m / z): $[\mathrm{M}+\mathrm{Na}]^{+}$calculated for $\mathrm{C}_{19} \mathrm{H}_{26} \mathrm{~N}_{2} \mathrm{O}_{3}: 353.1836$, found 353.1835. 


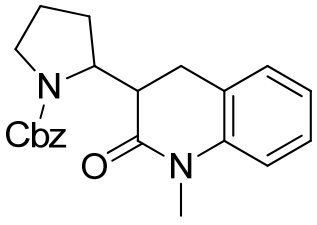

$( \pm)-7 a$

( $)$-Benzyl 2-(1-methyl-2-oxo-1,2,3,4-tetrahydroquinolin-3-yl)pyrrolidine-1-carboxylate (7a):

According to the general procedure, 1-methyl-2-quinolinone (3, $0.050 \mathrm{~g}, 0.314 \mathrm{mmol}), \mathrm{N}$ (benzyloxycarbonyl)-L-proline (6a, $0.117 \mathrm{~g}, 0471 \mathrm{mmol})$, cesium carbonate $(154 \mathrm{mg}, 0.471$ $\mathrm{mmol})$, and $\left[\operatorname{Ir}\left(\mathrm{dFCF}_{3} \text { ppy }\right)_{2}(\mathrm{dtbbpy})\right] \mathrm{PF}_{6}(\mathbf{1}, 3.5 \mathrm{mg}, 3.1 \mu \mathrm{mol})$ in DMSO $(15 \mathrm{~mL})$ were used, and the reaction was stirred for 1 day. Column chromatography afforded $83.4 \mathrm{mg}$ of 7a $(0.229$ mmol, $73 \%$ yield) as a yellow syrup.

${ }^{1} \mathbf{H}$ NMR $\left(400 \mathrm{MHz}, \mathrm{CDCl}_{3}\right) \delta: 7.63$ - $6.78(\mathrm{~m}, 9 \mathrm{H}), 5.23$ - $4.98(\mathrm{~m}, 2 \mathrm{H}), 4.67$ - $4.19(\mathrm{~m}, 1 \mathrm{H})$, $3.79-3.41(\mathrm{~m}, 2 \mathrm{H}), 3.38-3.25(\mathrm{~m}, 3 \mathrm{H}), 3.01-2.71(\mathrm{~m}, 2 \mathrm{H}), 2.46-2.14(\mathrm{~m}, 1 \mathrm{H}), 2.08-1.78(\mathrm{~m}$, $4 \mathrm{H})($ mixture of diastereomers and rotamers).

${ }^{13}$ C NMR (101 MHz, $\left.\mathrm{CDCl}_{3}\right) \delta: 170.76,170.57,155.43,154.74,140.17,140.04,136.86,136.59$, $130.78,129.47,128.59,128.50,128.34,128.13,128.08,127.92,127.81,127.76,127.62,127.43$, $127.26,125.73,125.01,122.71,122.53,122.02,114.37,114.07,67.88,66.75,66.62,66.51$, $58.04,56.82,56.33,47.71,47.01,46.51,43.69,43.29,42.50,39.66,32.58,29.61,29.46,28.81$, $28.42,28.23,27.46,25.98,25.54,24.16,23.76,23.62$ (mixture of diastereomers and rotamers).

FTIR (thin film) $v_{\max }: 3032,2951,2883,1694,1662,1601,1406,1355,1332,1264,1093,751$, $731,696 \mathrm{~cm}^{-1}$.

HRMS-ESI (m / z): $[\mathrm{M}+\mathrm{H}]^{+}$calculated for $\mathrm{C}_{22} \mathrm{H}_{24} \mathrm{~N}_{2} \mathrm{O}_{3}: 365.1860$, found 365.1862 . 


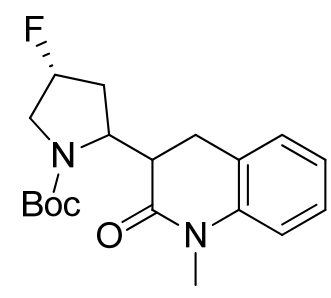

$7 b$

(4R)-tert-Butyl 4-fluoro-2-(1-methyl-2-oxo-1,2,3,4-tetrahydroquinolin-3-yl)pyrrolidine-1carboxylate (7b):

According to the general procedure, 1-methyl-2-quinolinone (3, $0.050 \mathrm{~g}, 0.314 \mathrm{mmol}), \mathrm{N}$-Boctrans-4-fluoro-L-proline $(\mathbf{6 b}, 0.110 \mathrm{~g}, 0.471 \mathrm{mmol})$, cesium carbonate $(154 \mathrm{mg}, 0.471 \mathrm{mmol})$, and $\left[\operatorname{Ir}\left(\mathrm{dFCF}_{3} \mathrm{ppy}\right)_{2}(\mathrm{dtbbpy}) \mathrm{PF}_{6}(\mathbf{1}, 3.5 \mathrm{mg}, 3.1 \mu \mathrm{mol})\right.$ in DMSO $(15 \mathrm{~mL})$ were used, and the reaction was stirred for 1 day. Column chromatography afforded $87.4 \mathrm{mg}$ of $7 \mathbf{b}(0.251 \mathrm{mmol}$, $80 \%$ yield) as a light yellow syrup.

${ }^{1} \mathbf{H}$ NMR $\left(500 \mathrm{MHz}, \mathrm{CDCl}_{3}\right) \delta: 7.31-7.20(\mathrm{~m}, 1 \mathrm{H}), 7.19-7.11(\mathrm{~m}, 1 \mathrm{H}), 7.07-6.89(\mathrm{~m}, 2 \mathrm{H})$, 5.35 - $5.05(\mathrm{~m}, 1 \mathrm{H}), 4.82$ - $4.43(\mathrm{~m}, 1 \mathrm{H}), 4.38$ - $3.82(\mathrm{~m}, 1 \mathrm{H}), 3.80$ - $3.65(\mathrm{~m}, 1 \mathrm{H}), 3.63$ - $3.38(\mathrm{~m}$, $1 \mathrm{H}), 3.32(\mathrm{~d}, J=18.9 \mathrm{~Hz}, 3 \mathrm{H}), 2.97-2.66(\mathrm{~m}, 2 \mathrm{H}), 2.58-2.13(\mathrm{~m}, 1 \mathrm{H}), 2.57-1.87(\mathrm{~m}, 2 \mathrm{H}), 1.53$ - $1.36(\mathrm{~m}, 9 \mathrm{H})$ (mixture of diastereomers and rotamers).

${ }^{13}$ C NMR (126 MHz, $\left.\mathrm{CDCl}_{3}\right) \delta: 171.29,170.37,170.24,154.91,154.56,140.14,140.04,139.95$, $139.89,127.90,127.85,127.63,127.57,127.34,127.27,126.29,125.60,122.96,122.76,122.62$, $114.45,114.39,114.33,93.77,93.54,92.40,92.14,80.31,79.76,79.56,60.26,56.86,56.61$, $56.48,55.38,54.13,53.94,53.58$ (q, $J=17.6 \mathrm{~Hz}, 1 \mathrm{C}), 44.85,44.38,42.42,41.93,41.64,39.51$, $39.36,37.78,34.84,34.66,34.51,29.64,29.40,29.36,29.27,29.20,28.79,28.65,28.47,28.37$, $28.32,28.31,28.16,25.28,24.19,20.94,14.10$ (mixture of diastereomers and rotamers).

${ }^{19}$ F NMR $\left(471 \mathrm{MHz}, \mathrm{CDCl}_{3}\right) \delta:-168.49(\mathrm{~s}, 1 \mathrm{~F}),-169.51(\mathrm{~s}, 1 \mathrm{~F}),-169.73(\mathrm{~s}, 1 \mathrm{~F}),-170.32(\mathrm{~s}$, $1 \mathrm{~F}),-175.27(\mathrm{~s}, 1 \mathrm{~F}),-176.72(\mathrm{~s}, 1 \mathrm{~F}),-177.39(\mathrm{~s}, 1 \mathrm{~F}),-178.06(\mathrm{~s}, 1 \mathrm{~F})$ (mixture of diastereomers and rotamers).

FTIR (thin film) $v_{\max }: 2973,2933,2887,1687,1662,1603,1390,1364,1265,1160,1119,753$, $732 \mathrm{~cm}^{-1}$.

HRMS-ESI (m / z): $[\mathrm{M}+\mathrm{H}]^{+}$calculated for $\mathrm{C}_{19} \mathrm{H}_{25} \mathrm{FN}_{2} \mathrm{O}_{3}: 349.1922$, found 349.1928. 
Determination of the diastereomeric ratio of $\mathbf{7 b}$ :<smiles>CN1C(=O)C(C2C[C@@H](F)CN2C(=O)OC(C)(C)C)Cc2ccccc21</smiles>

$7 b$

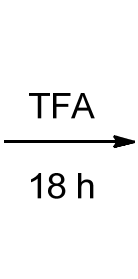

$8 \mathrm{~h}$<smiles>CN1C(=O)C(C2C[C@H](F)CN2)Cc2ccccc21</smiles>

S1 (TFA salt)

3-((4R)-4-Fluoropyrrolidin-2-yl)-1-methyl-3,4-dihydroquinolin-2(1H)-one 2,2,2trifluoroacetate (S1):

A TFA $(1 \mathrm{~mL})$ solution of $7 \mathbf{b}(0.067 \mathrm{~g}, 0.192 \mathrm{mmol})$ in a $40-\mathrm{mL}$ scintillation vial was stirred overnight $(18 \mathrm{~h})$ at room temperature. The sample was then concentrated under a stream of $\mathrm{N}_{2}$ and purified via reverse phase HPLC (5-95\% MeCN/water with $0.1 \%$ TFA) to afford $46.8 \mathrm{mg}$ of S1 (0.129 mmol, 67\% yield) as a white amorphous solid.

${ }^{1}$ H NMR (400 MHz, CD $\left.{ }_{3} \mathrm{CN}\right) \delta: 11.34-8.52(\mathrm{~m}, 1 \mathrm{H}), 7.35-7.29(\mathrm{~m}, 1 \mathrm{H}), 7.23(\mathrm{~d}, J=7.3 \mathrm{~Hz}$, $1 \mathrm{H}), 7.14$ - $7.04(\mathrm{~m}, 2 \mathrm{H}), 5.59-5.25(\mathrm{~m}, 1 \mathrm{H}), 4.18$ - $3.87(\mathrm{~m}, 1 \mathrm{H}), 3.77$ - $3.49(\mathrm{~m}, 2 \mathrm{H}), 3.48$ $3.41(\mathrm{~m}, 1 \mathrm{H}), 3.34-3.29(\mathrm{~m}, 3 \mathrm{H}), 3.25-2.99(\mathrm{~m}, 1 \mathrm{H}), 2.96-2.73(\mathrm{~m}, 2 \mathrm{H}), 2.39-2.05(\mathrm{~m}, 1 \mathrm{H})$ (mixture of diastereomers).

${ }^{13} \mathrm{C}$ NMR $\left(101 \mathrm{MHz}, \mathrm{CD}_{3} \mathrm{CN}\right) \delta: 171.60,171.46,171.20,161.91,161.56,141.04,140.95$, $140.70,140.63,129.24,129.18,126.01,125.94,124.82,124.71,124.64,116.61,116.59,94.57$, 93.77, 93.64, 93.47, 92.82, 92.03, 91.89, 91.72, 60.72, 60.52, 60.51, 60.02, 52.80, 52.96 (dd, $J=24.7,32.5 \mathrm{~Hz}, 1 \mathrm{C}$ ), 52.55, 43.34, 43.03, 40.69, 40.48, 37.68, 37.48, 36.02 (dd, $J=21.7,44.2$ $\mathrm{Hz}, 1 \mathrm{C}$ ), 35.49, 35.26, 30.81, 30.77, 30.62, 30.60, 29.71, 29.52, 29.43, 29.30 (mixture of diastereomers).

${ }^{19}$ F NMR (376 MHz, $\left.\mathrm{CD}_{3} \mathrm{CN}\right) \delta=-75.66(\mathrm{~s}, 3 \mathrm{~F}),-169.13(\mathrm{~s}, 1 \mathrm{~F}$, diastereomer 1), -172.08 (s, $1 \mathrm{~F}$, diastereomer 2), -173.02 (s, 1F, diastereomer 3), -173.20 (s, 1F, diastereomer 4).

The ratio of ${ }^{19} \mathrm{~F}$ NMR signals corresponding to the four diastereomers are 2.3:1.3:1.1:1.0.

FTIR (thin film) $v_{\max }$ : 2986(br), 2773(br), 1660, 1603, 1175, 1127, 757, $720 \mathrm{~cm}^{-1}$.

HRMS-ESI (m / z): [M+H] $]^{+}$calculated for $\mathrm{C}_{14} \mathrm{H}_{17} \mathrm{FN}_{2} \mathrm{O}$ : 249.1398, found 249.1402. 


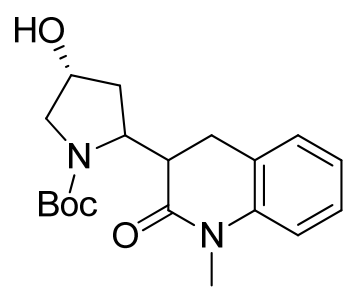

7c

(4R)-tert-Butyl 4-hydroxy-2-(1-methyl-2-oxo-1,2,3,4-tetrahydroquinolin-3-yl)pyrrolidine-1carboxylate (7c):

According to the general procedure, 1-methyl-2-quinolinone (3, $0.050 \mathrm{~g}, 0.314 \mathrm{mmol}), \mathrm{N}$-Boc-Lhydroxyproline $(6 \mathrm{c}, 109 \mathrm{mg}, 0.471 \mathrm{mmol})$, cesium carbonate $(154 \mathrm{mg}, 0.471 \mathrm{mmol})$, and $\left[\operatorname{Ir}\left(\mathrm{dFCF}_{3} \mathrm{ppy}_{2}(\mathrm{dtbbpy}) \mathrm{PF}_{6}(1,3.5 \mathrm{mg}, 3.1 \mu \mathrm{mol})\right.\right.$ in DMSO $(15 \mathrm{~mL})$ were used, and the reaction was stirred for 1 day. Column chromatography afforded $92.9 \mathrm{mg}$ of 7c $(0.268 \mathrm{mmol}$, $85 \%$ yield) as a white amorphous solid.

${ }^{1} \mathbf{H}$ NMR $\left(500 \mathrm{MHz}, \mathrm{CDCl}_{3}\right) \delta: 7.30-7.21(\mathrm{~m}, 1 \mathrm{H}), 7.15(\mathrm{~d}, J=6.7 \mathrm{~Hz}, 1 \mathrm{H}), 7.08-6.90(\mathrm{~m}, 2 \mathrm{H})$, 4.75 (br. s., 1H), 4.70 (br. s., $1 \mathrm{H}), 4.87$ - 4.19 (m, 2H), 3.83 - 3.39 (m, 2H), 3.36 - 3.27 (m, 3H), $3.06-2.67(\mathrm{~m}, 3 \mathrm{H}), 2.44-2.06(\mathrm{~m}, 1 \mathrm{H}), 1.99-1.65(\mathrm{~m}, 1 \mathrm{H}), 1.49$ - $1.36(\mathrm{~m}, 9 \mathrm{H}), 1.51$ - $1.34(\mathrm{~m}$, $10 \mathrm{H})$ (mixture of diastereomers and rotamers).

${ }^{13} \mathrm{C}$ NMR $\left(126 \mathrm{MHz}, \mathrm{CDCl}_{3}\right) \delta: 170.82,170.49,155.16,154.98,154.78,154.48,139.99,139.91$, $138.78,136.91,132.92$, 129.82, 128.12, 128.00, 127.89, 127.76, 127.46, 127.37, 125.66, 125.06, $122.97,122.76,122.43,120.76,114.57,114.42,114.39,114.07,80.10,79.50,79.25,70.46$, $69.74,69.17,60.35,56.90,56.73,55.63,55.23,42.48,41.86,37.27,36.68,36.58,36.31,34.91$, $31.80,30.02,29.77,29.52,29.11,28.94,28.72$, 28.55, 28.45, 28.41, 28.38, 28.32, 28.24, 26.57, $25.22,24.46,22.61,20.99,14.12,14.06,1.84$ (mixture of diastereomers and rotamers).

FTIR (thin film) $v_{\max }: 3423($ br), 2976, 2937, 1665, 1651, 1602, 1392, 1366, 1265, 1159, 1122 , $754,733 \mathrm{~cm}^{-1}$.

HRMS-ESI (m / z): [M+H] $]^{+}$calculated for $\mathrm{C}_{19} \mathrm{H}_{26} \mathrm{~N}_{2} \mathrm{O}_{4}: 347.1965$, found 347.1965. 
Determination of the diastereomeric ratio of $7 \mathrm{c}$ :
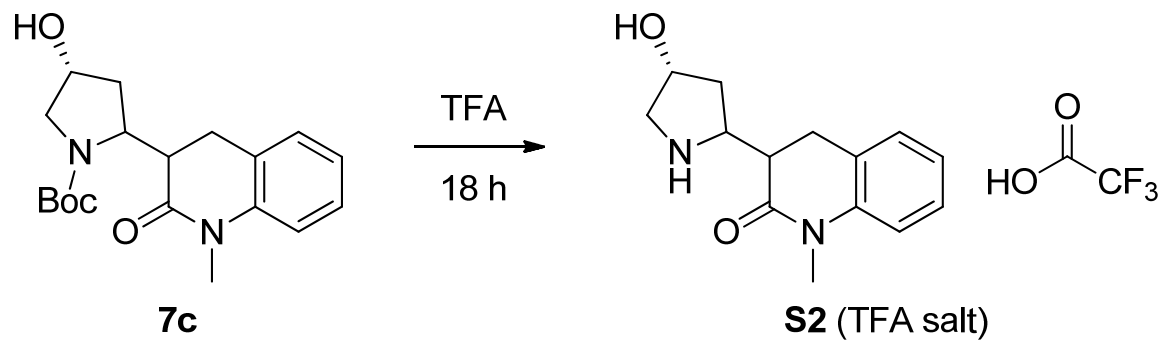

3-((4R)-4-Hydroxypyrrolidin-2-yl)-1-methyl-3,4-dihydroquinolin-2(1H)-one 2,2,2trifluoroacetate (S2):

A TFA ( $1 \mathrm{~mL})$ solution of 7c $(0.0929 \mathrm{~g}, 0.268 \mathrm{mmol})$ in a $40-\mathrm{mL}$ scintillation vial was stirred for $18 \mathrm{~h}$ at room temperature. The sample was then concentrated under a stream of $\mathrm{N}_{2}$ and purified via reverse phase HPLC (5-95\% MeCN/water with $0.1 \%$ TFA) to afford $18.3 \mathrm{mg}$ of S2 $(0.0508$ mmol, $19 \%$ yield) as a white amorphous solid.

${ }^{1}$ H NMR $\left(400 \mathrm{MHz}, \mathrm{CD}_{3} \mathrm{CN}\right) \delta: 7.31-7.22(\mathrm{~m}, 1 \mathrm{H}), 7.22-7.16(\mathrm{~m}, 1 \mathrm{H}), 7.08-6.96(\mathrm{~m}, 2 \mathrm{H})$, $4.28-4.22(\mathrm{~m}, 1 \mathrm{H}), 4.21-4.15(\mathrm{~m}, 1 \mathrm{H}), 4.21(\mathrm{~s}, 1 \mathrm{H}), 3.57-3.54(\mathrm{~m}, 1 \mathrm{H}), 3.53-3.47(\mathrm{~m}, 1 \mathrm{H})$, 3.47 - 3.40 (m, 1H), 3.48 (br. s., $1 \mathrm{H}), 3.28(\mathrm{~d}, J=2.3 \mathrm{~Hz}, 3 \mathrm{H}), 3.23$ - $3.08(\mathrm{~m}, 1 \mathrm{H}), 3.02$ - 2.89 (m, 2H), $2.86-2.66(\mathrm{~m}, 2 \mathrm{H}), 2.59$ (ddd, $J=5.3,7.8,10.8 \mathrm{~Hz}, 1 \mathrm{H}$ ), 2.18 (ddd, $J=6.7,7.8,13.3 \mathrm{~Hz}$, $1 \mathrm{H}), 2.24-2.05(\mathrm{~m}, 1 \mathrm{H}), 2.14-2.05(\mathrm{~m}, 1 \mathrm{H}), 1.82-1.75(\mathrm{~m}, 1 \mathrm{H}), 1.74-1.68(\mathrm{~m}, 1 \mathrm{H}), 1.83-$ $1.68(\mathrm{~m}, 1 \mathrm{H}), 1.61(\mathrm{ddd}, J=6.0,9.8,13.0 \mathrm{~Hz}, 1 \mathrm{H}), 1.55-1.50(\mathrm{~m}, 1 \mathrm{H}), 1.50-1.41(\mathrm{~m}, 1 \mathrm{H}), 1.65$ - $1.41(\mathrm{~m}, 1 \mathrm{H})$ (mixture of diastereomers).

Analysis of the carbinol methine and pyrrolidine-2-methine revealed an approximate diastereomeric ratio of 1.4:1.4:1.0:1.0.

${ }^{13} \mathrm{C}$ NMR $\left(101 \mathrm{MHz}, \mathrm{CD}_{3} \mathrm{CN}\right) \delta: 172.75,172.48,141.82,141.78,141.69,129.43,129.34$, $129.13,128.71,128.68,128.56,127.24,127.17,124.01,123.90,123.89,116.04,115.98,115.83$, $73.63,73.46,72.41,59.29,58.31,56.78,56.66,56.39,56.21,47.26,47.11,46.95,40.74,40.55$, $40.45,30.42,30.34,29.94,29.59,29.19,28.78$ (mixture of diastereomers).

FTIR (thin film) $v_{\max }: 3332$ (br), 2955, 2923, 2853, 1652, 1601, 1464, 1373, 1266, 1126, 755 $\mathrm{cm}^{-1}$.

HRMS-ESI (m / z): $[\mathrm{M}+\mathrm{H}]^{+}$calculated for $\mathrm{C}_{14} \mathrm{H}_{18} \mathrm{~N}_{2} \mathrm{O}_{2}: 247.1441$, found 247.1444. 


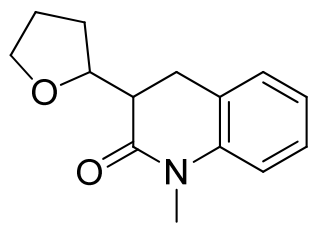

$( \pm)-7 d$

\section{( \pm )-1-Methyl-3-(tetrahydrofuran-2-yl)-3,4-dihydroquinolin-2(1H)-one (7d):}

According to the general procedure, 1-methyl-2-quinolinone (3, $0.050 \mathrm{~g}, 0.314 \mathrm{mmol}),( \pm)-1$ tetrahydro-2-furoic acid (6d, $0.055 \mathrm{~g}, 0.471 \mathrm{mmol})$, cesium carbonate $(154 \mathrm{mg}, 0.471 \mathrm{mmol})$, and $\left[\operatorname{Ir}\left(\mathrm{dFCF}_{3} \text { ppy }\right)_{2}(\mathrm{dtbbpy})\right] \mathrm{PF}_{6}(1,3.5 \mathrm{mg}, 3.1 \mu \mathrm{mol})$ in DMSO $(15 \mathrm{~mL})$ were used, and the reaction was stirred for 1 day. Column chromatography afforded $52.8 \mathrm{mg}$ of $7 \mathbf{d}(0.228 \mathrm{mmol}$, $73 \%$ yield) as a colorless syrup.

${ }^{1}$ H NMR $\left(500 \mathrm{MHz}, \mathrm{CDCl}_{3}\right) \delta: 6.75-6.69(\mathrm{~m}, 1 \mathrm{H}), 6.69-6.60(\mathrm{~m}, 1 \mathrm{H}), 6.47(\mathrm{q}, \mathrm{J}=6.8 \mathrm{~Hz}, 1 \mathrm{H})$, $6.42(\mathrm{dd}, J=3.4,8.0 \mathrm{~Hz}, 1 \mathrm{H}), 3.76-3.48(\mathrm{~m}, 1 \mathrm{H}), 3.47-3.24(\mathrm{~m}, 1 \mathrm{H}), 3.24-3.12(\mathrm{~m}, 1 \mathrm{H}), 2.82$ $(\mathrm{d}, J=3.9 \mathrm{~Hz}, 3 \mathrm{H}), 2.58-2.27(\mathrm{~m}, 2 \mathrm{H}), 2.28-1.95(\mathrm{~m}, 1 \mathrm{H}), 1.65-1.46(\mathrm{~m}, 1 \mathrm{H}), 1.44-1.09(\mathrm{~m}$, $3 \mathrm{H})$ (mixture of diastereomers).

${ }^{13}$ C NMR (126 MHz, $\left.\mathrm{CDCl}_{3}\right) \delta: 170.43,140.02,128.20,127.88,127.38,127.27,125.48,125.16$, $122.89,122.75,114.44,114.39$, 77.45, 76.52, 68.36, 67.86, 46.00, 44.82, 30.22, 29.72, 29.57, $28.11,27.51,26.59,25.82,25.78$ (mixture of diastereomers).

FTIR (thin film) $v_{\max }: 2956,2924,2873,2854,1666,1602,1462,1365,1262,1128,1067,754$ $\mathrm{cm}^{-1}$.

HRMS-ESI (m / z): $[\mathrm{M}+\mathrm{Na}]^{+}$calculated for $\mathrm{C}_{14} \mathrm{H}_{17} \mathrm{NO}_{2}: 254.1151$, found 254.1153. 


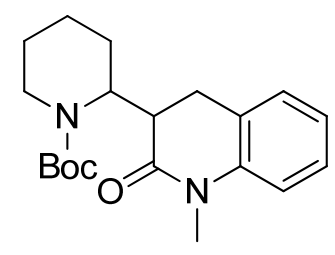

$( \pm)-7 e$

( $)$-tert-Butyl 2-(1-methyl-2-oxo-1,2,3,4-tetrahydroquinolin-3-yl)piperidine-1-carboxylate (7e):

According to the general procedure, 1-methyl-2-quinolinone (3,0.050 g, $0.314 \mathrm{mmol}),( \pm)-1-$ (tert-butoxycarbonyl)piperidine-2-carboxylic acid $(\mathbf{6 e}, 0.108 \mathrm{~g}, 0.471 \mathrm{mmol})$, cesium carbonate (154 $\mathrm{mg}, 0.471 \mathrm{mmol})$, and $\left[\operatorname{Ir}\left(\mathrm{dFCF}_{3} \text { ppy }\right)_{2}(\mathrm{dtbbpy})\right] \mathrm{PF}_{6}(1,3.5 \mathrm{mg}, 3.1 \mu \mathrm{mol})$ in DMSO (15 $\mathrm{mL}$ ) were used, and the reaction was stirred for $3 \mathrm{~h}$. Column chromatography afforded $100.5 \mathrm{mg}$ of 7 e $(0.292 \mathrm{mmol}, 93 \%$ yield $)$ as a yellow amorphous solid.

${ }^{1} \mathbf{H}$ NMR $\left(400 \mathrm{MHz}, \mathrm{CDCl}_{3}\right) \delta: 7.30$ - $7.23(\mathrm{~m}, 1 \mathrm{H}), 7.19-7.08(\mathrm{~m}, 1 \mathrm{H}), 7.07$ - $6.94(\mathrm{~m}, 2 \mathrm{H})$, 4.44 - $3.92(\mathrm{~m}, 2 \mathrm{H}), 3.44-3.27(\mathrm{~m}, 3 \mathrm{H}), 3.25-3.10(\mathrm{~m}, 2 \mathrm{H}), 2.97-2.60(\mathrm{~m}, 2 \mathrm{H}), 1.92$ - $1.69(\mathrm{~m}$, $2 \mathrm{H}), 1.64-1.56(\mathrm{~m}, 2 \mathrm{H}), 1.48-1.40(\mathrm{~m}, 2 \mathrm{H}), 1.38-1.22(\mathrm{~m}, 9 \mathrm{H})$ (mixture of diastereomers and rotamers).

${ }^{13}$ C NMR (101 MHz, $\left.\mathrm{CDCl}_{3}\right) \delta: 170.73,170.27,154.43,140.28,128.80,128.04,127.71,127.39$, $124.91,123.36,123.04,122.56,114.53,114.45,79.52$, 78.92, 49.65, 39.73, 39.49, 38.75, 29.87, $29.66,29.41,28.46,28.34,28.11,27.61,27.45,25.26,19.18,18.78$ (mixture of diastereomers and rotamers).

FTIR (thin film) $v_{\max }: 2972,2931,2867,1666,1602,1412,1364,1262,1161,1116,751,731$ $\mathrm{cm}^{-1}$.

HRMS-ESI (m / z): $[\mathrm{M}+\mathrm{H}]^{+}$calculated for $\mathrm{C}_{20} \mathrm{H}_{28} \mathrm{~N}_{2} \mathrm{O}_{3}: 345.2173$, found 345.2171 . 


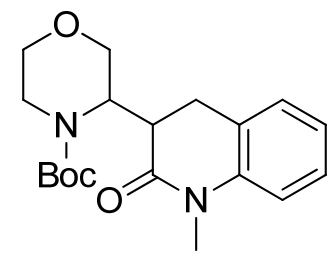

$( \pm)-7 f$

( \pm -tert-Butyl 3-(1-methyl-2-oxo-1,2,3,4-tetrahydroquinolin-3-yl)morpholine-4-carboxylate (7f):

According to the general procedure, 1-methyl-2-quinolinone (3, $0.050 \mathrm{~g}, 0.314 \mathrm{mmol}),( \pm)-4-$ (tert-butoxycarbonyl)morpholine-3-carboxylic acid $(\mathbf{6 f}, 0.109 \mathrm{~g}, 0.471 \mathrm{mmol})$, cesium carbonate (154 mg, $0.471 \mathrm{mmol})$, and $\left[\operatorname{Ir}\left(\mathrm{dFCF}_{3} \text { ppy }\right)_{2}(\mathrm{dtbbpy})\right] \mathrm{PF}_{6}(\mathbf{1}, 3.5 \mathrm{mg}, 3.1 \mu \mathrm{mol})$ in DMSO (15 $\mathrm{mL}$ ) were used, and the reaction was stirred for $15 \mathrm{~h}$. Column chromatography afforded 105.3 $\mathrm{mg}$ of $\mathbf{7 f}(0.292 \mathrm{mmol}, 97 \%$ yield $)$ as a yellow syrup.

${ }^{1} \mathbf{H}$ NMR (400 MHz, $\left.\mathrm{CDCl}_{3}\right) \delta: 7.31-7.23(\mathrm{~m}, 1 \mathrm{H}), 7.19-7.09(\mathrm{~m}, 1 \mathrm{H}), 7.06-6.94(\mathrm{~m}, 2 \mathrm{H})$, 4.36 - $4.18(\mathrm{~m}, 1 \mathrm{H}), 4.41(\mathrm{~d}, J=10.4 \mathrm{~Hz}, 1 \mathrm{H}), 4.05-3.88(\mathrm{~m}, 1 \mathrm{H}), 3.87-3.75(\mathrm{~m}, 1 \mathrm{H}), 3.68$ $3.54(\mathrm{~m}, 1 \mathrm{H}), 3.50-3.39(\mathrm{~m}, 2 \mathrm{H}), 3.39-3.27(\mathrm{~m}, 3 \mathrm{H}), 3.69$ (br. s., $8 \mathrm{H}), 3.26-3.15(\mathrm{~m}, 1 \mathrm{H})$, 3.69 - $3.14(\mathrm{~m}, 8 \mathrm{H}), 2.96-2.63(\mathrm{~m}, 2 \mathrm{H}), 3.06-2.56(\mathrm{~m}, 2 \mathrm{H}), 3.09-2.55(\mathrm{~m}, 2 \mathrm{H}), 1.51-1.31(\mathrm{~m}$, 9H) (mixture of diastereomers and rotamers).

${ }^{13}$ C NMR (101 MHz, $\left.\mathrm{CDCl}_{3}\right) \delta: 170.88,154.71,140.16,140.10,128.01,127.90,127.80,127.61$, $127.50,125.49,122.97,122.69,114.62,114.56,80.38,79.65,69.58,69.28,66.98,66.74,66.53$, $66.24,51.16,49.60,40.82,39.20,37.79,37.56,29.87,29.62,28.25,28.23,27.36$ (mixture of diastereomers and rotamers).

FTIR (thin film) $v_{\max }: 2972,2927,2858,1691,1666,1410,1365,1296,1266,1167,1118,754$ $\mathrm{cm}^{-1}$.

HRMS-ESI (m / z): [M+Na $]^{+}$calculated for $\mathrm{C}_{19} \mathrm{H}_{26} \mathrm{~N}_{2} \mathrm{O}_{4}: 369.1785$, found 369.1786 . 


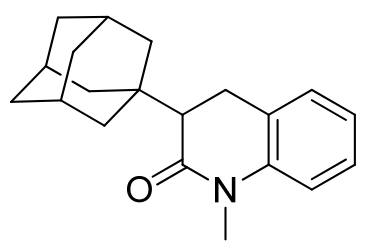

$( \pm)-7 g$

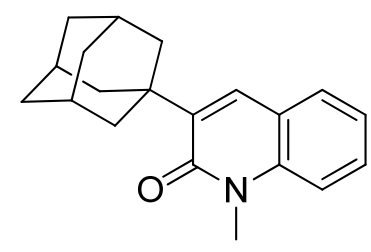

$( \pm)-\mathbf{S 3}$

( \pm )-3-(Adamantan-1-yl)-1-methyl-3,4-dihydroquinolin-2(1H)-one $(7 \mathrm{~g})$ and $( \pm)-3-$ (adamantan-1-yl)-1-methylquinolin-2(1H)-one (S3):

According to the general procedure, 1-methyl-2-quinolinone (3, $0.050 \mathrm{~g}, 0.314 \mathrm{mmol}), 1$ adamantanecarboxylic acid $(6 \mathrm{~g}, 0.085 \mathrm{~g}, 0.471 \mathrm{mmol})$, cesium carbonate $(154 \mathrm{mg}, 0.471 \mathrm{mmol})$, and $\left[\operatorname{Ir}\left(\mathrm{dFCF}_{3} \text { ppy }\right)_{2}(\mathrm{dtbbpy})\right] \mathrm{PF}_{6}(\mathbf{1}, 3.5 \mathrm{mg}, 3.1 \mu \mathrm{mol})$ in DMSO $(15 \mathrm{~mL})$ were used, and the reaction was stirred for 1 day. Column chromatography afforded $75.4 \mathrm{mg}$ of an inseperable 2:1 mixture of $\mathbf{7 g}$ and $\mathbf{S 3}(0.255 \mathrm{mmol}, 81 \%$ yield $)$ as a yellow syrup.

${ }^{1} \mathrm{H}$ NMR $\left(400 \mathrm{MHz}, \mathrm{CDCl}_{3}\right) \delta: 7.54(\mathrm{dd}, J=1.6,7.8 \mathrm{~Hz}, 1 \mathrm{H}, \mathrm{S} 3), 7.52-7.47$ (m, 2H, S3), 7.31 (d, $J=8.3 \mathrm{~Hz}, 1 \mathrm{H}, \mathbf{S 3}), 7.25-7.20(\mathrm{~m}, 1 \mathrm{H}$, both $\mathbf{7 g}$ and S3), $7.21-7.17(\mathrm{~m}, 1 \mathrm{H}, \mathbf{7 g}), 7.14(\mathrm{~d}, J=7.3$ $\mathrm{Hz}, 1 \mathrm{H}, 7 \mathbf{g}), 7.02-6.96(\mathrm{~m}, 1 \mathrm{H}, 7 \mathrm{~g}), 6.91(\mathrm{dd}, J=0.6,8.1 \mathrm{~Hz}, 1 \mathrm{H}), 3.71$ (s, 3H, S3), 3.38 (s, 3H, $7 \mathbf{g}), 3.10-3.02(\mathrm{~m}, 1 \mathrm{H}, 7 \mathbf{g}), 3.01-2.95(\mathrm{~m}, 1 \mathrm{H}, 7 \mathbf{g}), 2.29(\mathrm{dd}, J=3.5,6.4 \mathrm{~Hz}, 1 \mathrm{H}, 7 \mathrm{~g}), 2.16(\mathrm{~m}$, $2 \mathrm{H}$, both $\mathbf{7 g}$ and $\mathbf{S 3}), 1.94-1.76(\mathrm{~m}, 5 \mathrm{H}$, both $\mathbf{7 g}$ and $\mathbf{S 3}), 1.63-1.47(\mathrm{~m}, 8 \mathrm{H}$, both $\mathbf{7 g}$ and $\mathbf{S 3})$.

${ }^{13}$ C NMR (101 MHz, $\left.\mathrm{CDCl}_{3}\right) \delta: 170.82,161.37,140.71,138.85,133.48,129.33,128.54,127.27$, $127.05,126.45,122.75,121.63,120.59,114.11,113.46,51.28,40.54,39.85,38.62,37.50,37.00$, $36.70,36.40,35.86,29.56,29.37,28.78,28.74,28.63,27.82,26.50$ (mixture of $\mathbf{7 g}$ and S3).

FTIR (thin film) $v_{\max }: 2902,2848,1639,1593,1453,1346,1234,1105,751,735 \mathrm{~cm}^{-1}$.

7g: HRMS-ESI (m / z): [M+Na] $]^{+}$calculated for $\mathrm{C}_{20} \mathrm{H}_{25} \mathrm{NO}$ : 318.1828 , found 318.1824 .

S3: HRMS-ESI (m / z): $[\mathrm{M}+\mathrm{H}]^{+}$calculated for $\mathrm{C}_{20} \mathrm{H}_{23} \mathrm{NO}$ : 294.1852, found 294.1855. 


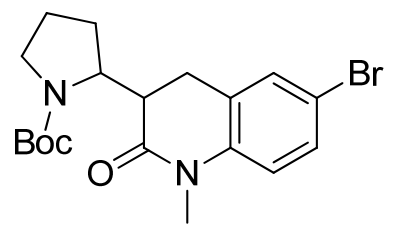

$( \pm)-9 a$

(土)-tert-Butyl 2-(6-bromo-1-methyl-2-oxo-1,2,3,4-tetrahydroquinolin-3-yl)pyrrolidine-1carboxylate (9a):

According to the general procedure, 6-bromo-1-methylquinolin-2(1H)-one (8a, $0.075 \mathrm{~g}, 0.314$ $\mathrm{mmol}), \mathrm{N}$-(tert-butoxycarbonyl)-L-proline $(2,0.101 \mathrm{~g}, 0.471 \mathrm{mmol})$, cesium carbonate (154 $\mathrm{mg}$, $0.471 \mathrm{mmol})$, and $\left[\operatorname{Ir}\left(\mathrm{dFCF}_{3} \mathrm{ppy}\right)_{2}(\mathrm{dtbbpy})\right] \mathrm{PF}_{6}(\mathbf{1}, 3.5 \mathrm{mg}, 3.1 \mu \mathrm{mol})$ in DMSO $(15 \mathrm{~mL})$ were used, and the reaction was stirred for 1 day. Column chromatography afforded $65.9 \mathrm{mg}$ of 9a (0.161 mmol, 51\% yield) as a light yellow syrup.

${ }^{1}$ H NMR (400 MHz, $\left.\mathrm{CDCl}_{3}\right)$ \&: 7.36 (br. s., 1H), 7.29 (d, J=2.1 Hz, 1H), 6.89 - 6.77 (m, 1H), $4.64-4.08(\mathrm{~m}, 1 \mathrm{H}), 3.70-3.37(\mathrm{~m}, 1 \mathrm{H}), 3.70-3.34(\mathrm{~m}, 2 \mathrm{H}), 3.34-3.26(\mathrm{~m}, 3 \mathrm{H}), 3.18(\mathrm{dt}$, $J=5.5,8.8 \mathrm{~Hz}, 1 \mathrm{H}), 2.94-2.71(\mathrm{~m}, 2 \mathrm{H}), 2.32-1.94(\mathrm{~m}, 1 \mathrm{H}), 1.90-1.69(\mathrm{~m}, 3 \mathrm{H}), 1.48$ - 1.37 (m, 9H) (mixture of diastereomers and rotamers).

${ }^{13}$ C NMR $\left(101 \mathrm{MHz}, \mathrm{CDCl}_{3}\right) \delta: 170.50,155.12,154.56,139.35,130.78,130.67,130.28,130.10$, $128.07,127.11,115.96,115.38,115.06,79.83,79.24,57.36,56.52,56.03,47.04,46.71,43.82$, $42.84,29.73,29.67,29.56,28.63,28.50,28.47,28.13,27.66,26.22,25.08,24.01,23.80,22.66$ (mixture of diastereomers and rotamers).

FTIR (thin film) $v_{\max }: 2972,2928,2885,1685,1672,1491,1389,1364,1350,1258,1160,1122$, $1095,808,733 \mathrm{~cm}^{-1}$.

HRMS-ESI (m / z): $[\mathrm{M}+\mathrm{H}]^{+}$calculated for $\mathrm{C}_{19} \mathrm{H}_{25} \mathrm{BrN}_{2} \mathrm{O}_{3}: 409.1121$, found 409.1118 . 


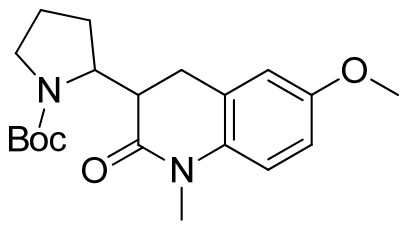

$( \pm)-9 b$

( \pm )-tert-Butyl 2-(6-methoxy-1-methyl-2-oxo-1,2,3,4-tetrahydroquinolin-3-yl)pyrrolidine-1carboxylate (9b):

According to the general procedure, 6-methoxy-1-methylquinolin-2(1H)-one $(\mathbf{8 b}, 0.059 \mathrm{~g}, 0.314$ $\mathrm{mmol}), \mathrm{N}$-(tert-butoxycarbonyl)-L-proline $(2,0.101 \mathrm{~g}, 0.471 \mathrm{mmol})$, cesium carbonate (154 $\mathrm{mg}$, $0.471 \mathrm{mmol})$, and $\left[\operatorname{Ir}\left(\mathrm{dFCF}_{3} \mathrm{ppy}\right)_{2}(\mathrm{dtbbpy})\right] \mathrm{PF}_{6}(\mathbf{1}, 3.5 \mathrm{mg}, 3.1 \mu \mathrm{mol})$ in DMSO $(15 \mathrm{~mL})$ were used, and the reaction was stirred for 1 day. Column chromatography afforded $73.8 \mathrm{mg}$ of $\mathbf{9 b}$ (0.205 mmol, 65\% yield) as a yellow amorphous solid.

${ }^{1}$ H NMR (400 MHz, $\left.\mathrm{CDCl}_{3}\right) \delta: 6.94-6.81(\mathrm{~m}, 1 \mathrm{H}), 6.80-6.68(\mathrm{~m}, 2 \mathrm{H}), 4.62-4.09(\mathrm{~m}, 1 \mathrm{H})$, 3.77 (s, 3H), 3.30 (br. s., 5H), $2.92-2.65$ (m, 2H), $2.51-2.05$ (m, 1H), $2.02-1.70$ (m, 4H), 1.47 - $1.33(\mathrm{~m}, 9 \mathrm{H})$ (mixture of diastereomers and rotamers).

${ }^{13}$ C NMR $\left(101 \mathrm{MHz}, \mathrm{CDCl}_{3}\right) \delta: 170.98,170.31,155.31,155.14,155.06,154.59,133.78,133.63$, 127.36, 126.77, 115.17, 113.81, 112.24, 111.84, 111.64, 79.62, 78.97, 60.25, 57.38, 56.56, 56.22, $55.78,55.41,55.39,55.30,47.54,47.21,46.53,45.99,44.17,43.96,43.05,42.65,32.40,31.76$, $29.45,28.90,28.60,28.41,28.35,28.20,28.11,27.50,26.37,25.52,24.05,23.74,23.27,22.57$, 22.30, 20.92 (mixture of diastereomers and rotamers).

FTIR (thin film) $v_{\max }: 2968,2936,2887,1686,1659,1504,1389,1364,1238,1160,1112,1030$, $731 \mathrm{~cm}^{-1}$.

HRMS-ESI (m / z): $[\mathrm{M}+\mathrm{H}]^{+}$calculated for $\mathrm{C}_{20} \mathrm{H}_{28} \mathrm{~N}_{2} \mathrm{O}_{4}: 361.2122$, found 361.2127. 


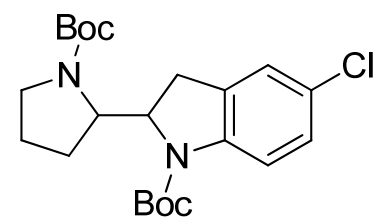

$( \pm)-9 c$

(士)-tert-Butyl 2-(1-(tert-butoxycarbonyl)pyrrolidin-2-yl)-5-chloroindoline-1-carboxylate (9c):

According to the general procedure, 1-Boc-5-chloroindole (8c, $0.079 \mathrm{~g}, 0.314 \mathrm{mmol}), \mathrm{N}$-(tertbutoxycarbonyl)-L-proline (2, $0.101 \mathrm{~g}, 0.471 \mathrm{mmol})$, cesium carbonate $(154 \mathrm{mg}, 0.471 \mathrm{mmol})$, and $\left[\operatorname{Ir}\left(\mathrm{dFCF}_{3} \text { ppy }\right)_{2}(\mathrm{dtbbpy})\right] \mathrm{PF}_{6}(1,3.5 \mathrm{mg}, 3.1 \mu \mathrm{mol})$ in DMSO $(15 \mathrm{~mL})$ were used, and the reaction was stirred for 2 days. Column chromatography afforded $131.0 \mathrm{mg}$ of $\mathbf{9 c}(0.310 \mathrm{mmol}$, $99 \%$ yield) as a colorless syrup.

${ }^{1} \mathbf{H}$ NMR $\left(500 \mathrm{MHz}, \mathrm{CDCl}_{3}\right) \delta: 7.89-7.34(\mathrm{~m}, 1 \mathrm{H}), 7.15-6.99(\mathrm{~m}, 2 \mathrm{H}), 5.00-4.50(\mathrm{~m}, 1 \mathrm{H})$, $4.28-3.86(\mathrm{~m}, 1 \mathrm{H}), 3.70-3.36(\mathrm{~m}, 1 \mathrm{H}), 3.33-3.10(\mathrm{~m}, 3 \mathrm{H}), 2.87-2.60(\mathrm{~m}, 1 \mathrm{H}), 1.85$ - $1.74(\mathrm{~m}$, $3 \mathrm{H}), 1.52(\mathrm{~d}, J=5.4 \mathrm{~Hz}, 9 \mathrm{H}), 1.43-1.31(\mathrm{~m}, 9 \mathrm{H})($ mixture of diastereomers and rotamers).

${ }^{13}$ C NMR (126 MHz, $\left.\mathrm{CDCl}_{3}\right) \delta: 154.91,154.53,154.42,152.59,152.20,141.58,133.02,132.07$, $127.49,127.11,126.90,124.57,124.45,122.59,122.36,116.64,116.29,81.11,79.44,78.70$, $61.28,60.46,58.66,47.60,46.72,45.64,31.73,30.30,29.56,28.57,28.42,28.35,28.30,28.26$, $28.22,28.05,27.92,27.48,26.34,25.92,25.25,24.10,23.68,23.12,22.75,22.55$ (mixture of diastereomers and rotamers).

FTIR (thin film) $v_{\max }: 2975,2931,2878,1688,1477,1384,1365,1161,1137,1018,731 \mathrm{~cm}^{-1}$.

HRMS-ESI (m / z): [M+H] $]^{+}$calculated for $\mathrm{C}_{22} \mathrm{H}_{31} \mathrm{ClN}_{2} \mathrm{O}_{4}: 423.2045$, found 423.2048 . 


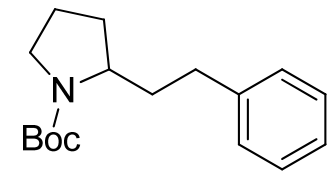

$( \pm)-9 d$

\section{( \pm )-tert-Butyl 2-phenethylpyrrolidine-1-carboxylate (9d):}

According to the general procedure, styrene (8d, $0.033 \mathrm{~g}, 0.314 \mathrm{mmol}), N$-(tert-butoxycarbonyl)L-proline $(2,0.101 \mathrm{~g}, 0.471 \mathrm{mmol})$, cesium carbonate $(154 \mathrm{mg}, 0.471 \mathrm{mmol})$, and $\left[\operatorname{Ir}\left(\mathrm{dFCF}_{3} \mathrm{ppy}_{2}(\mathrm{dtbbpy}) \mathrm{PF}_{6}(\mathbf{1}, 3.5 \mathrm{mg}, 3.1 \mu \mathrm{mol})\right.\right.$ in DMSO $(15 \mathrm{~mL})$ were used, and the reaction was stirred for $5 \mathrm{~h}$. Column chromatography afforded $66.4 \mathrm{mg}$ of $9 d(0.241 \mathrm{mmol}, 77 \%$ yield) as a light yellow syrup.

${ }^{1}$ H NMR (400 MHz, $\left.\mathrm{CDCl}_{3}\right)$ \&: 7.33 - 7.25 (m, 2H), 7.24 - 7.15 (m, 3H), 3.81 (br. s., 1H), 3.55 $3.23(\mathrm{~m}, 2 \mathrm{H}), 2.80-2.51(\mathrm{~m}, 2 \mathrm{H}), 2.08$ (br. s., $1 \mathrm{H}), 2.03-1.78(\mathrm{~m}, 3 \mathrm{H}), 1.77$ - $1.68(\mathrm{~m}, 1 \mathrm{H})$, $1.68-1.57(\mathrm{~m}, 1 \mathrm{H}), 1.52-1.43(\mathrm{~m}, 9 \mathrm{H})$.

${ }^{13} \mathrm{C}$ NMR $\left(101 \mathrm{MHz}, \mathrm{CDCl}_{3}\right) \delta: 154.55,141.98,128.25,128.23,125.65,78.88,56.95,46.20$, $36.20,32.74,30.38,28.49,23.22$.

FTIR (thin film) $v_{\max }: 2971,2929,2873,1687,1389,1363,1166,1097,698 \mathrm{~cm}^{-1}$.

HRMS-ESI (m / z): $[\mathrm{M}+\mathrm{H}]^{+}$calculated for $\mathrm{C}_{17} \mathrm{H}_{25} \mathrm{NO}_{2}: 276.1958$, found 276.1953. 


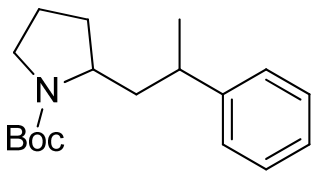

$( \pm)-9 e$

( $)$-tert-Butyl 2-(2-phenylpropyl)pyrrolidine-1-carboxylate (9e):

According to the general procedure, $\alpha$-methylstyrene $(\mathbf{8 e}, 0.037 \mathrm{~g}, 0.314 \mathrm{mmol}), N$-(tertbutoxycarbonyl)-L-proline (2, $0.101 \mathrm{~g}, 0.471 \mathrm{mmol})$, cesium carbonate $(154 \mathrm{mg}, 0.471 \mathrm{mmol})$, and $\left[\operatorname{Ir}\left(\mathrm{dFCF}_{3} \text { ppy }\right)_{2}(\mathrm{dtbbpy})\right] \mathrm{PF}_{6}(\mathbf{1}, 3.5 \mathrm{mg}, 3.1 \mu \mathrm{mol})$ in DMSO $(15 \mathrm{~mL})$ were used, and the reaction was stirred for $5 \mathrm{~h}$. Column chromatography afforded $71.9 \mathrm{mg}$ of $\mathbf{9 e}(0.248 \mathrm{mmol}, 79 \%$ yield) as a colorless syrup.

${ }^{1}$ H NMR $\left(400 \mathrm{MHz}, \mathrm{CDCl}_{3}\right) \delta: 7.33-7.26(\mathrm{~m}, 2 \mathrm{H}), 7.26-7.16(\mathrm{~m}, 3 \mathrm{H}), 3.95-3.47(\mathrm{~m}, 1 \mathrm{H})$, $3.44-3.22(\mathrm{~m}, 2 \mathrm{H}), 2.82-2.63(\mathrm{~m}, 1 \mathrm{H}), 2.31-1.98(\mathrm{~m}, 1 \mathrm{H}), 1.93-1.66(\mathrm{~m}, 4 \mathrm{H}), 1.61-1.52(\mathrm{~m}$, $1 \mathrm{H}), 1.51-1.42(\mathrm{~m}, 9 \mathrm{H}), 1.29$ (dd, $J=6.8,16.0 \mathrm{~Hz}, 3 \mathrm{H})$ (mixture of diastereomers).

${ }^{13}$ C NMR (101 MHz, $\left.\mathrm{CDCl}_{3}\right) \delta: 154.51,154.38,147.95,146.19,128.34,128.32,127.02,126.69$, $125.94,125.87,78.97,56.21,55.29,46.02,42.87,41.92,37.63,37.38,30.11,28.55,23.81$, 23.14, 21.49 (mixture of diastereomers).

FTIR (thin film) $v_{\max }: 2965,2929,2873,1687,1389,1364,1163,1105,762,694 \mathrm{~cm}^{-1}$.

HRMS-ESI (m / z): $[\mathrm{M}+\mathrm{H}]^{+}$calculated for $\mathrm{C}_{18} \mathrm{H}_{27} \mathrm{NO}_{2}: 290.2115$, found 290.2115 . 


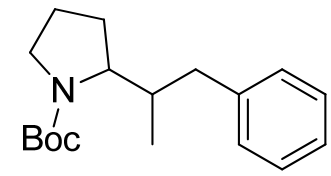

$( \pm)-9 f$

\section{( \pm -tert-Butyl 2-(1-phenylpropan-2-yl)pyrrolidine-1-carboxylate (9f):}

According to the general procedure, trans- $\beta$-methylstyrene (8f, $0.037 \mathrm{~g}, 0.314 \mathrm{mmol}), N$-(tertbutoxycarbonyl)-L-proline $(2,0.101 \mathrm{~g}, 0.471 \mathrm{mmol})$, cesium carbonate $(154 \mathrm{mg}, 0.471 \mathrm{mmol})$, and $\left[\operatorname{Ir}\left(\mathrm{dFCF}_{3} \text { ppy }\right)_{2}(\mathrm{dtbbpy})\right] \mathrm{PF}_{6}(\mathbf{1}, 3.5 \mathrm{mg}, 3.1 \mu \mathrm{mol})$ in DMSO $(15 \mathrm{~mL})$ were used, and the reaction was stirred for 1 day. Column chromatography afforded $48.2 \mathrm{mg}$ of 9 f $(0.167 \mathrm{mmol}$, $53 \%$ yield) as a colorless syrup.

${ }^{1}$ H NMR $\left(500 \mathrm{MHz}, \mathrm{CDCl}_{3}\right) \delta: 7.31-7.25$ (m, 2H), 7.18 (br. s., 2H), 7.14 (d, J=7.3 Hz, 1H), 3.95 - $3.69(\mathrm{~m}, 1 \mathrm{H}), 3.67$ - $3.47(\mathrm{~m}, 1 \mathrm{H}), 3.33$ - $3.18(\mathrm{~m}, 1 \mathrm{H}), 2.82$ - $2.54(\mathrm{~m}, 1 \mathrm{H}), 2.53$ - $2.33(\mathrm{~m}$, $1 \mathrm{H}), 2.30-2.10(\mathrm{~m}, 1 \mathrm{H}), 1.99-1.71(\mathrm{~m}, 4 \mathrm{H}), 1.53-1.45(\mathrm{~m}, 9 \mathrm{H}), 0.79(\mathrm{~d}, J=7.0 \mathrm{~Hz}, 3 \mathrm{H})$ (mixture of diastereomers and rotamers).

${ }^{13} \mathrm{C}$ NMR (126 MHz, $\left.\mathrm{CDCl}_{3}\right) \delta: 154.82,141.40,141.01,129.01,128.91,128.15,128.10,125.68$, $125.58,79.04,62.06,60.44,47.05,40.63,37.69,28.52,27.18,25.93,24.31,23.87,16.02,13.65$ (mixture of diastereomers and rotamers).

FTIR (thin film) $v_{\max }: 2969,2930,2874,1687,1390,1364,1162,1104,699 \mathrm{~cm}^{-1}$.

HRMS-ESI (m / z): [M+Na $]^{+}$calculated for $\mathrm{C}_{18} \mathrm{H}_{27} \mathrm{NO}_{2}$ : 312.1934, found 312.1931. 
<smiles>COC(=O)C(Cc1ccccc1)C1CCCN1C(=O)OCc1ccccc1</smiles>

$( \pm)-9 g$<smiles>COC(=O)CC(c1ccccc1)C1CCCN1C(=O)OCc1ccccc1</smiles>

$( \pm)-\mathbf{S 4}$

( \pm )-tert-Butyl 2-(1-methoxy-1-oxo-3-phenylpropan-2-yl)pyrrolidine-1-carboxylate (9g) and ( \pm )-tert-butyl 2-(3-methoxy-3-oxo-1-phenylpropyl)pyrrolidine-1-carboxylate (S4):

According to the general procedure, methyl cinnamate $(\mathbf{8 g}, 0.051 \mathrm{~g}, 0.314 \mathrm{mmol}), \mathrm{N}$-(tertbutoxycarbonyl)-L-proline (2, $0.101 \mathrm{~g}, 0.471 \mathrm{mmol})$, cesium carbonate (154 $\mathrm{mg}, 0.471 \mathrm{mmol})$, and $\left[\operatorname{Ir}\left(\mathrm{dFCF}_{3} \text { ppy }\right)_{2}(\mathrm{dtbbpy})\right] \mathrm{PF}_{6}(\mathbf{1}, 3.5 \mathrm{mg}, 3.1 \mu \mathrm{mol})$ in DMSO $(15 \mathrm{~mL})$ were used, and the reaction was stirred for $5 \mathrm{~h}$. Column chromatography afforded $81.2 \mathrm{mg}$ of an inseperable mixture of $\mathbf{9 g}$ and S4 (0.244 mmol, $78 \%$ yield $)$ as a colorless syrup.

${ }^{1} \mathbf{H}$ NMR (400 MHz, $\left.\mathrm{CDCl}_{3}\right) \delta: 7.34$ - $7.10(\mathrm{~m}, 5 \mathrm{H}), 4.28$ - $3.87(\mathrm{~m}, 1 \mathrm{H}), 3.60$ - $3.52(\mathrm{~m}, 3 \mathrm{H})$, $3.51-3.37(\mathrm{~m}, 1 \mathrm{H}), 3.37-3.23(\mathrm{~m}, 1 \mathrm{H}), 3.22-3.06(\mathrm{~m}, 1 \mathrm{H}), 2.97(\mathrm{dd}, J=11.2,13.5 \mathrm{~Hz}, 1 \mathrm{H})$, $2.81-2.61(\mathrm{~m}, 1 \mathrm{H}), 2.12-1.87(\mathrm{~m}, 2 \mathrm{H}), 1.85-1.70(\mathrm{~m}, 2 \mathrm{H}), 1.52-1.43(\mathrm{~m}, 9 \mathrm{H})$ (mixture of regioisomers, diastereomers, and rotamers).

${ }^{13}$ C NMR (101 MHz, $\left.\mathrm{CDCl}_{3}\right) \delta: 174.08,173.56,154.38,140.01,139.06,128.74,128.64,128.34$, $128.20,128.08,126.80,126.65,126.26,126.10,125.95,79.75,79.65,79.14,61.63,61.54,58.94$, $58.60,51.48,51.40,50.78,49.72,49.10,47.19,46.71,46.58,46.29,46.10,45.61,37.78,35.71$, $35.45,31.95,31.80,29.59,29.21,28.47,28.44,28.40,28.18,27.94,27.43,27.54,24.01,23.67$, $23.12,22.95,22.58,22.26$ (mixture of regioisomers, diastereomers, and rotamers).

FTIR (thin film) $v_{\max }: 2973,2933,2880,1732,1688,1389,1365,1160,1113,699 \mathrm{~cm}^{-1}$.

HRMS-ESI (m / z): [M+Na $]^{+}$calculated for $\mathrm{C}_{19} \mathrm{H}_{27} \mathrm{NO}_{4}: 356.1832$, found 156.1833. 
Determination of the regioisomeric ratio of $\mathbf{9 g}$ and $\mathbf{S 4}$ :<smiles>COC(=O)C(Cc1ccccc1)C1CCCN1C(=O)OCc1ccccc1</smiles>

$( \pm)-9 g$<smiles>COC(=O)CC(c1ccccc1)C1CCCN1C(=O)OCc1ccccc1</smiles>

$( \pm)-\mathbf{S 4}$

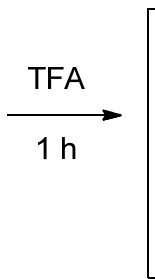

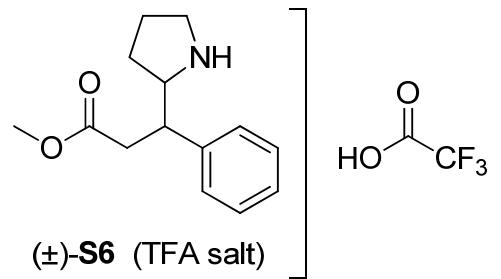

( \pm )-Methyl 3-phenyl-2-(pyrrolidin-2-yl)propanoate 2,2,2-trifluoroacetate (S5) and ( \pm )methyl 3-phenyl-3-(pyrrolidin-2-yl)propanoate 2,2,2-trifluoroacetate (S6):

A TFA (1 mL) solution of the mixture containing $9 \mathrm{~g}$ and $\mathbf{S} 4(81 \mathrm{mg}, 0.243 \mathrm{mmol})$ in a $40-\mathrm{mL}$ scintillation vial was stirred at $\mathrm{rt}$ for $1 \mathrm{~h}$. The TFA was then removed under a stream of $\mathrm{N}_{2}$, and the yellow residue was purified via column chromatography (25 g Biotage Snap Ultra column, 0$100 \%$ EtOAc/hept then $0-100 \% \mathrm{CH}_{2} \mathrm{Cl}_{2} / \mathrm{MeOH}$ ) to afford $70.2 \mathrm{mg}$ of of an inseperable 5.7:1 mixture of S5 and S6 (0.202 mmol, 83\% yield) as a colorless syrup.

${ }^{1} \mathbf{H}$ NMR (500 MHz, $\left.\mathrm{CDCl}_{3}\right) \delta$ : $10.41-9.89$ (m, 1H, both S5 and S6), $9.60-9.14$ (m, 1H, both S5 and S6), $7.34-7.15(\mathrm{~m}, 3 \mathrm{H}$, both S5 and S6), $7.10(\mathrm{dd}, J=7.1,15.7 \mathrm{~Hz}, 2 \mathrm{H}, \mathrm{S5}), 3.756$ (m, 1H, S6), 3.698 (m, 1H, S6), 3.58 (s, 3H, S5 diastereomer 1), 3.51 (s, 3H, S5 diastereomer 2), 3.46 (s, 3H, S6), $3.43-3.25(\mathrm{~m}, 2 \mathrm{H}$, both S5 and S6), $3.24-3.11(\mathrm{~m}, 1 \mathrm{H}, \mathbf{S 6}), 3.10-3.01(\mathrm{~m}$, 2H, both S5 and S6), $2.99-2.88$ (m, 1H, S5), 2.77 (dd, J=9.3, $16.1 \mathrm{~Hz}, 1 \mathrm{H}, \mathbf{S 6}), 2.21$ (m, 2H, S20), 2.16 - 2.01 (m, 1H, both S5 and S6), $2.01-1.91(\mathrm{~m}, 1 \mathrm{H}$, both S5 and S6), $1.89-1.71(\mathrm{~m}$, $1 \mathrm{H}$, both S5 and S6), $1.67(\mathrm{~m}, 1 \mathrm{H}, \mathrm{S6}), 1.63-1.53(\mathrm{~m}, 1 \mathrm{H}, \mathrm{S6})$ (mixture of regioisomers and diastereomers).

${ }^{13} \mathrm{C}$ NMR $\left(151 \mathrm{MHz}, \mathrm{CDCl}_{3}\right) \delta: 173.02,172.14,171.93,162.21\left(\mathrm{q}, J_{\mathrm{C}-\mathrm{F}}=35.4 \mathrm{~Hz}\right), 139.67$, $136.96,136.47,128.89,128.62,128.57,128.48,127.64,127.56,127.03,126.79,116.45\left(\mathrm{q}, J_{\mathrm{C}-}\right.$ $\mathrm{F}=292.3 \mathrm{~Hz}), 64.25,60.62,59.84,52.17,51.89,51.69,49.46,48.77,45.37,45.28,45.14,44.43$, $38.76,36.11,35.85,30.18,29.28,28.68,23.59,23.57,23.18$ (mixture of regioisomers and diastereomers).

FTIR (thin film) $v_{\max }$ : 2955(br), 2773(br), 1732, 1666, 1436, 1172, 1128, 833, 798, 720, 701 $\mathrm{cm}^{-1}$.

HRMS-ESI (m / z): [M+H $]^{+}$calculated for $\mathrm{C}_{14} \mathrm{H}_{19} \mathrm{NO}_{2}: 234.1489$, found 234.1492.

2D NMR spectroscopic analysis of the mixture of S5 and S6 indicated that the major product was regioisomer S5, and the minor product regioisomer S6. Key HMBC correlations are shown below:

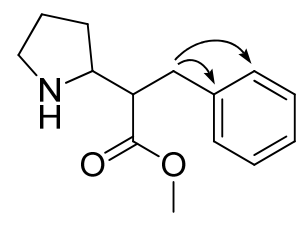

$( \pm)-\mathbf{S 5}$<smiles>COC(=O)CC1(C2CCCC2)CC2c3ccccc3C21</smiles>

$( \pm)-\mathbf{S 6}$ 


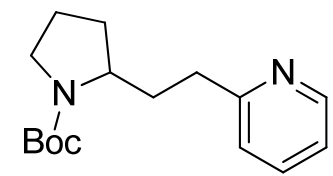

$( \pm)-9 h$

( \pm )-tert-Butyl 2-(2-(pyridin-2-yl)ethyl)pyrrolidine-1-carboxylate (9h):

According to the general procedure, 2-vinylpyridine (8h, $0.033 \mathrm{~g}, 0.314 \mathrm{mmol}), \mathrm{N}$-(tertbutoxycarbonyl)-L-proline $(2,0.101 \mathrm{~g}, 0.471 \mathrm{mmol})$, cesium carbonate (154 $\mathrm{mg}, 0.471 \mathrm{mmol})$, and $\left[\operatorname{Ir}\left(\mathrm{dFCF}_{3} \text { ppy }\right)_{2}(\mathrm{dtbbpy})\right] \mathrm{PF}_{6}(\mathbf{1}, 3.5 \mathrm{mg}, 3.1 \mu \mathrm{mol})$ in DMSO $(15 \mathrm{~mL})$ were used, and the reaction was stirred for $5 \mathrm{~h}$. Column chromatography afforded $50.0 \mathrm{mg}$ of $\mathbf{9 h}(0.181 \mathrm{mmol}, 58 \%$ yield) as a yellow syrup.

${ }^{1}$ H NMR (400 MHz, $\left.\mathrm{CDCl}_{3}\right) \delta: 8.47(\mathrm{~d}, J=3.1 \mathrm{~Hz}, 1 \mathrm{H}), 7.60-7.48(\mathrm{~m}, 1 \mathrm{H}), 7.15(\mathrm{~d}, J=16.0 \mathrm{~Hz}$, 1H), 7.06 (br. s., 1H), $3.99-3.67(\mathrm{~m}, 1 \mathrm{H}), 3.47$ - 3.21 (m, 2H), 2.86 - 2.63 (m, 2H), $2.24-2.01$ $(\mathrm{m}, 1 \mathrm{H}), 2.00-1.82(\mathrm{~m}, 2 \mathrm{H}), 1.81-1.64(\mathrm{~m}, 3 \mathrm{H}), 1.41(\mathrm{~s}, 9 \mathrm{H})$ (mixture of rotamers).

${ }^{13}$ C NMR (101 MHz, $\left.\mathrm{CDCl}_{3}\right) \delta: 161.70,154.57,149.11,148.98,136.26,122.52,120.89,78.93$, $57.06,56.84,46.42,46.03,35.25,34.73,34.15,30.56,29.92,28.47,23.71,23.01$ (mixture of rotamers).

FTIR (thin film) $v_{\max }: 2970,2930,2872,1686,1389,1364,1166,1102,769,748 \mathrm{~cm}^{-1}$.

HRMS-ESI (m / z): [M+H] $]^{+}$calculated for $\mathrm{C}_{16} \mathrm{H}_{24} \mathrm{~N}_{2} \mathrm{O}_{2}: 277.1911$, found 277.1912. 


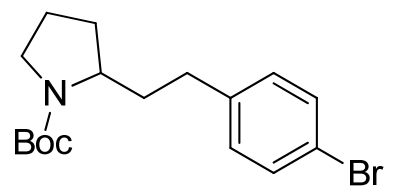

$( \pm)-9 i$

\section{( \pm )-tert-Butyl 2-(4-bromophenethyl)pyrrolidine-1-carboxylate (9i):}

According to the general procedure, 1-bromo-4-vinylbenzene (8i, $0.058 \mathrm{~g}, 0.314 \mathrm{mmol}), \mathrm{N}$-(tertbutoxycarbonyl)-L-proline $(2,0.101 \mathrm{~g}, 0.471 \mathrm{mmol})$, cesium carbonate $(154 \mathrm{mg}, 0.471 \mathrm{mmol})$, and $\left[\operatorname{Ir}\left(\mathrm{dFCF}_{3} \text { ppy }\right)_{2}(\mathrm{dtbbpy})\right] \mathrm{PF}_{6}(\mathbf{1}, 3.5 \mathrm{mg}, 3.1 \mu \mathrm{mol})$ in DMSO $(15 \mathrm{~mL})$ were used, and the reaction was stirred for $5 \mathrm{~h}$. Column chromatography afforded $71.1 \mathrm{mg}$ of $9 \mathrm{i}(0.201 \mathrm{mmol}, 64 \%$ yield) as a light yellow syrup.

${ }^{1}$ H NMR (500 MHz, $\mathrm{CDCl}_{3}$ ) $\delta: 7.49$ - 7.32 (m, 2H), 7.05 (br. s., 1H), 7.21 - 6.95 (m, 2H), 3.95 $3.67(\mathrm{~m}, 1 \mathrm{H}), 3.49-3.22(\mathrm{~m}, 2 \mathrm{H}), 2.65-2.42(\mathrm{~m}, 1 \mathrm{H}), 2.20-1.89(\mathrm{~m}, 2 \mathrm{H}), 1.88-1.74(\mathrm{~m}, 2 \mathrm{H})$, $1.74-1.53(\mathrm{~m}, 2 \mathrm{H}), 1.51-1.39(\mathrm{~m}, 9 \mathrm{H})$ (mixture of rotamers).

${ }^{13} \mathrm{C}$ NMR $\left(126 \mathrm{MHz}, \mathrm{CDCl}_{3}\right) \delta: 154.58,154.50,141.01,140.79,131.28,129.99,128.21,119.37$, $79.01,78.82,78.75,56.96,56.59,46.45,46.05,45.85,45.52,36.10,35.66,32.09,30.50,29.94$, $28.46,25.66,24.91,23.75,23.04,22.60$ (mixture of rotamers).

FTIR (thin film) $v_{\max }: 2971,2929,2873,1687,1390,1364,1167,1103 \mathrm{~cm}^{-1}$.

HRMS-ESI (m / z): [M-t-Bu+H] $]^{+}$calculated for $\mathrm{C}_{17} \mathrm{H}_{24} \mathrm{BrNO}_{2}$ : 298.0437, found 298.0445. 
<smiles>C=Cc1ccccc1N</smiles>

18<smiles>COc1ccc(C(=O)Cl)cc1</smiles>

19<smiles>C=Cc1ccccc1NC(=O)c1ccc(OC)cc1</smiles>

20

\section{4-Methoxy- $N$-(2-vinylphenyl)benzamide (20):}

A $\mathrm{CH}_{2} \mathrm{Cl}_{2}(20 \mathrm{~mL})$ solution of 2-vinylaniline $(18,0.475 \mathrm{~g}, 3.99 \mathrm{mmol})$ and triethylamine $(0.484$ $\mathrm{g}, 4.78 \mathrm{mmol})$ in a $25-\mathrm{mL}$ round-bottom flask was treated with $p$-anisoyl chloride $(\mathbf{1 9}, 1.02 \mathrm{~g}$, $5.98 \mathrm{mmol})$. The colorless solution was stirred overnight $(18 \mathrm{~h})$ at $\mathrm{rt}$. The reaction was quenched with water and extracted thrice with $\mathrm{CH}_{2} \mathrm{Cl}_{2}$. The organic layers were combined, filtered through a phase separator, and concentrated in vacuo to a white amorphous solid. Column chromatography (25 g Biotage Snap Ultra column, 0-100\% EtOAc/hept with 5\% $\mathrm{CH}_{2} \mathrm{Cl}_{2}$ ) afforded $0.785 \mathrm{~g}$ of $\mathbf{2 0}$ ( $3.10 \mathrm{mmol}, 78 \%$ yield) as a white amorphous solid.

${ }^{1}$ H NMR (500 MHz, $d_{6}$-DMSO) $\delta: 9.90(\mathrm{~s}, 1 \mathrm{H}), 8.10-7.95(\mathrm{~m}, 2 \mathrm{H}), 7.68(\mathrm{~d}, J=7.5 \mathrm{~Hz}, 1 \mathrm{H})$, $7.34-7.22(\mathrm{~m}, 3 \mathrm{H}), 7.19-7.01(\mathrm{~m}, 2 \mathrm{H}), 6.85(\mathrm{dd}, J=11.2,17.6 \mathrm{~Hz}, 1 \mathrm{H}), 5.80(\mathrm{dd}, J=1.2,17.5$ $\mathrm{Hz}, 1 \mathrm{H}), 5.86-5.74(\mathrm{~m}, 1 \mathrm{H}), 5.28(\mathrm{dd}, J=1.2,11.0 \mathrm{~Hz}, 1 \mathrm{H}), 3.93-3.81(\mathrm{~m}, 3 \mathrm{H}), 3.92-3.81(\mathrm{~m}$, $3 \mathrm{H})$ (mixture of rotamers).

${ }^{13}$ C NMR (126 MHz, DMSO) $\delta: 165.06,161.87,135.40,133.44,132.69,132.67,129.53$, $127.99,127.87,126.34,126.25,125.24,115.28,114.65,113.58,55.40$ (mixture of rotamers).

FTIR (thin film) $v_{\max }: 3270(\mathrm{br}), 1643,1606,1501,1252,1175,765 \mathrm{~cm}^{-1}$.

HRMS-ESI (m / z): $[\mathrm{M}+\mathrm{H}]^{+}$calculated for $\mathrm{C}_{16} \mathrm{H}_{15} \mathrm{NO}_{2}:$ 254.1176, found 254.1179. 

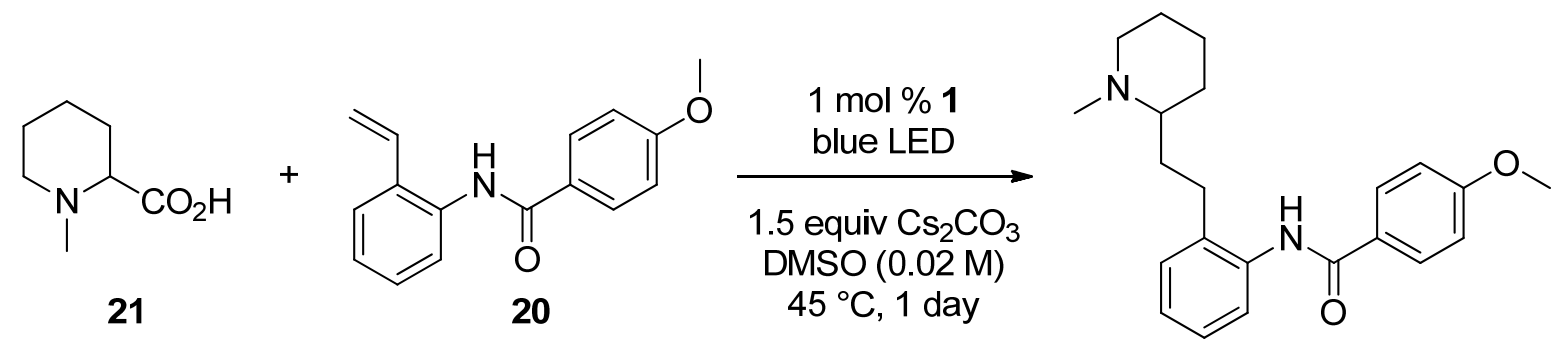

(士)-17 (encainide)

\section{( \pm )-4-Methoxy- $N$-(2-(2-(1-methylpiperidin-2-yl)ethyl)phenyl)benzamide (17, encainide):}

According to the general procedure, $20(0.100 \mathrm{~g}, 0.395 \mathrm{mmol}), N$-methyl DL-pipecolic acid (21, $0.085 \mathrm{~g}, 0.592 \mathrm{mmol})$, cesium carbonate $(193 \mathrm{mg}, 0.592 \mathrm{mmol})$, and $\left[\operatorname{Ir}\left(\mathrm{dFCF}_{3} \text { ppy }\right)_{2}(\mathrm{dtbbpy})\right] \mathrm{PF}_{6}$ $(1,4.4 \mathrm{mg}, 4.0 \mu \mathrm{mol})$ in DMSO $(20 \mathrm{~mL})$ were used, and the reaction was stirred for 1 day. Column chromatography (25 g Biotage Snap Ultra column, 0-100\% EtOAc/heptane then 0-80\% $\mathrm{CH}_{2} \mathrm{Cl}_{2} / \mathrm{MeOH}$ afforded $98.0 \mathrm{mg}$ of $17(0.278 \mathrm{mmol}, 70 \%$ yield $)$ as a white amorphous solid.

${ }^{1} \mathbf{H}$ NMR $\left(500 \mathrm{MHz}, \mathrm{CDCl}_{3}\right) \delta: 10.71$ (br. s., $\left.1 \mathrm{H}\right), 8.14(\mathrm{~d}, J=8.0 \mathrm{~Hz}, 1 \mathrm{H}), 7.86(\mathrm{~d}, J=8.6 \mathrm{~Hz}$, 2H), $7.28-7.23(\mathrm{~m}, 1 \mathrm{H}), 7.20(\mathrm{dd}, J=1.3,7.5 \mathrm{~Hz}, 1 \mathrm{H}), 7.13-7.09(\mathrm{~m}, 1 \mathrm{H}), 6.96(\mathrm{~d}, J=8.6 \mathrm{~Hz}$, 2H), $3.87(\mathrm{~s}, 3 \mathrm{H}), 2.88-2.76(\mathrm{~m}, 1 \mathrm{H}), 2.69(\mathrm{td}, J=5.4,14.1 \mathrm{~Hz}, 1 \mathrm{H}), 2.44(\mathrm{~d}, J=12.7 \mathrm{~Hz}, 1 \mathrm{H})$, 2.33 - $2.22(\mathrm{~m}, 4 \mathrm{H}), 2.22-2.15(\mathrm{~m}, 1 \mathrm{H}), 1.94-1.83(\mathrm{~m}, 1 \mathrm{H}), 1.73(\mathrm{dq}, J=4.3,9.6 \mathrm{~Hz}, 1 \mathrm{H}), 1.66$ $1.56(\mathrm{~m}, 1 \mathrm{H}), 1.52-1.39(\mathrm{~m}, 1 \mathrm{H}), 1.35-1.16(\mathrm{~m}, 3 \mathrm{H}), 1.14-1.07(\mathrm{~m}, 1 \mathrm{H})$.

${ }^{13}$ C NMR $\left(126 \mathrm{MHz}, \mathrm{CDCl}_{3}\right) \delta: 166.74,161.83,137.32,133.42,129.75,129.27,128.81,126.42$, 124.64, 123.77, 113.34, 58.61, 55.34, 54.94, 36.76, 34.82, 26.51, 26.09, 24.49, 20.89.

FTIR (thin film) $v_{\max }: 3230(\mathrm{br}), 2930,2854,2779,1665,1607,1505,1448,1301,1250,1173$, $1027,842,761 \mathrm{~cm}^{-1}$.

HRMS-ESI (m / z): [M+H] $]^{+}$calculated for $\mathrm{C}_{22} \mathrm{H}_{28} \mathrm{~N}_{2} \mathrm{O}_{2}: 353.2224$, found 353.2232. 


\section{Determination of Addition Regioselectivity.}

Using the general procedure, $\mathrm{N}$-(tert-butoxycarbonyl)glycine and cyclohexanecarboxylic acid were both coupled to 1-methyl-2-quinolinone (3) to afford S7 and S8, respectively.<smiles>CN1C(=O)C(CNC(=O)OC(C)(C)C)Cc2ccccc21</smiles>

( \pm -S7<smiles>CN1C(=O)C(C2CCCCC2)Cc2ccccc21</smiles>

$( \pm)-\mathbf{S} 8$

2D NMR spectroscopic analyses of these compounds were more straightforward than analyses of other products containing diastereomeric mixtures of rotamers. Key HMBC (black) and ROESY (blue) correlations to establish connectivity are shown below.

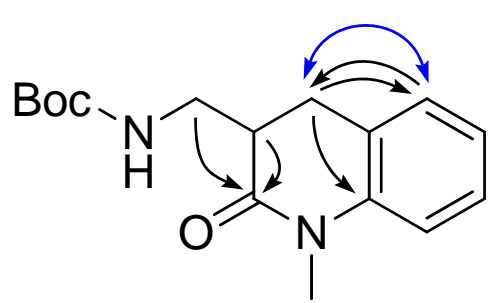

$( \pm)-S 7$

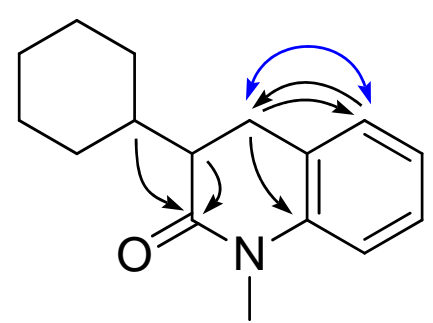

( \pm -S8 


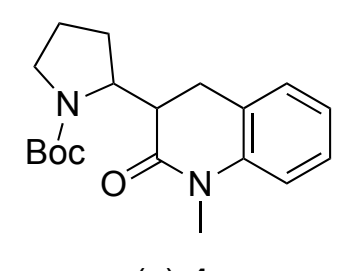

( \pm -4
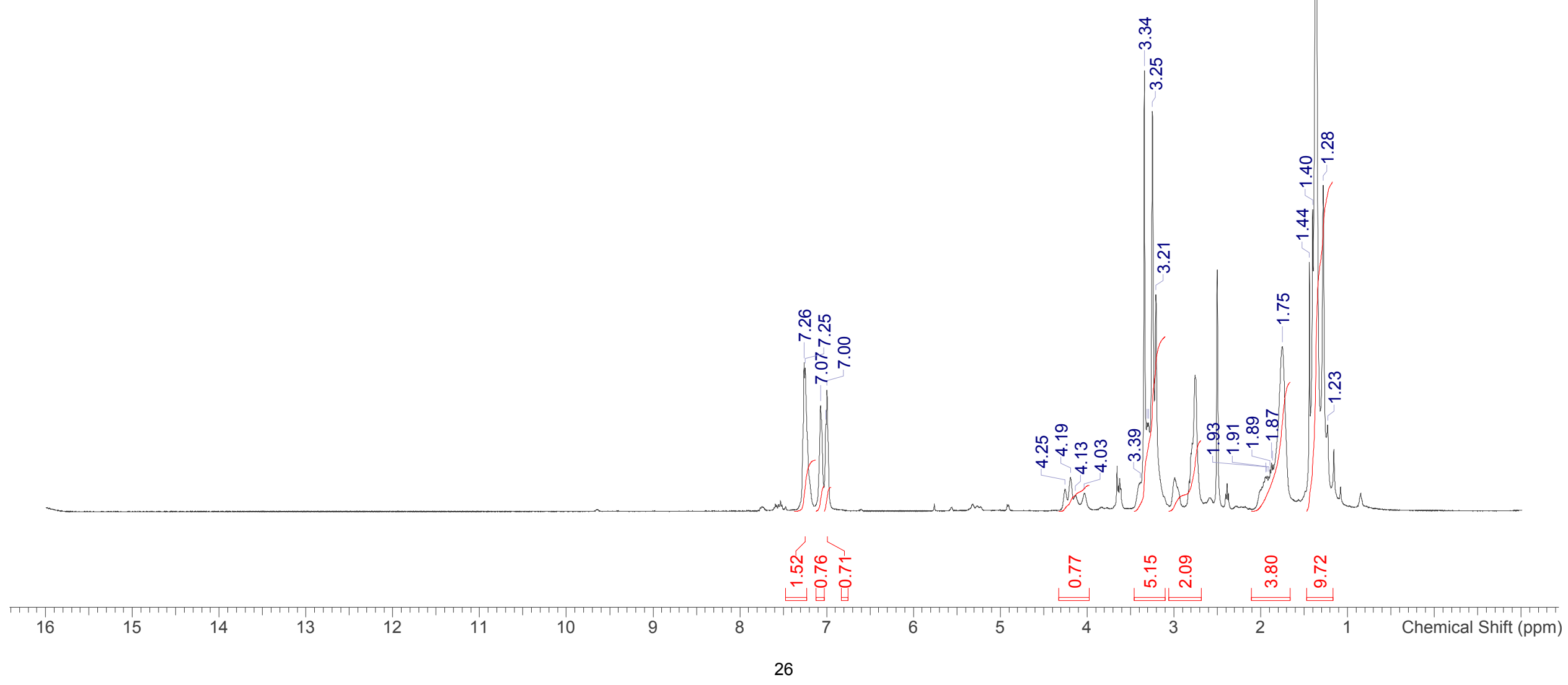


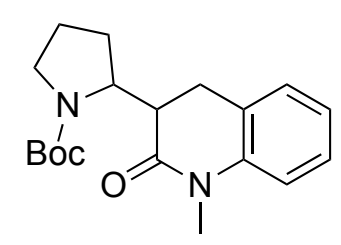

$( \pm)-4$

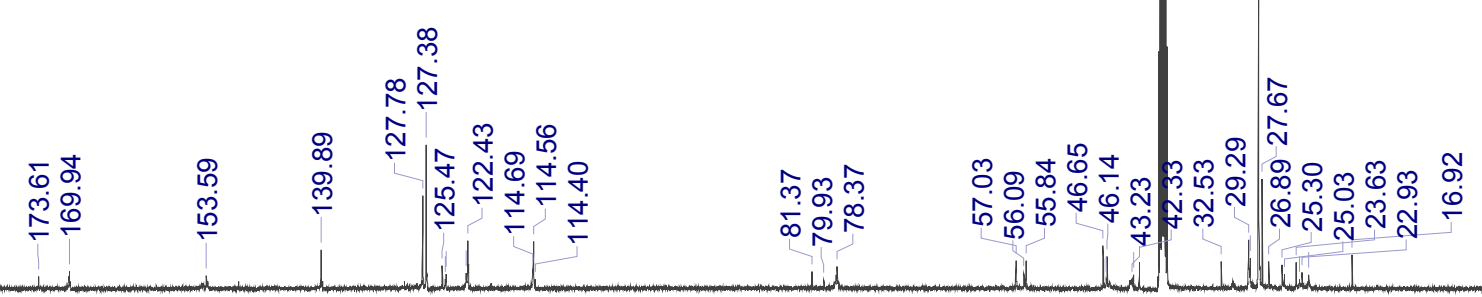




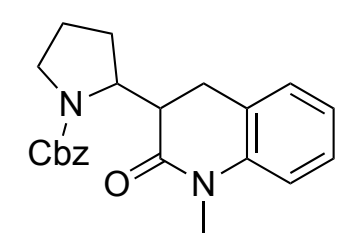

( \pm -7a
N

$\stackrel{\Re}{N}$

ల్ల

$\stackrel{\infty}{\sim}$

is

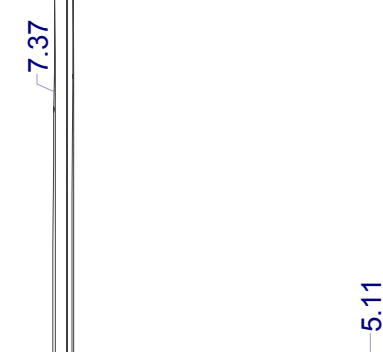

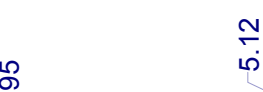

i⿱

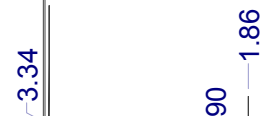

0

ח.

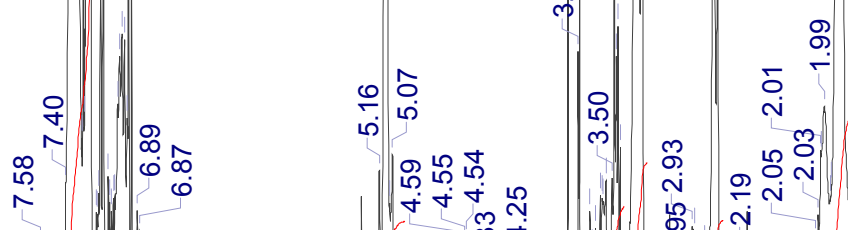

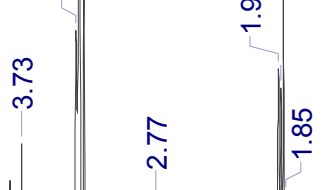

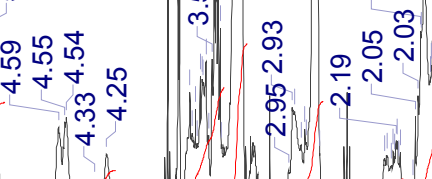

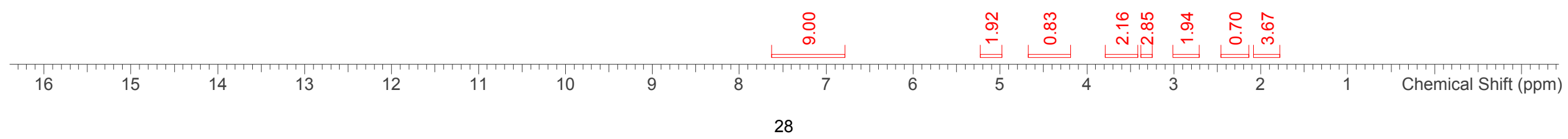




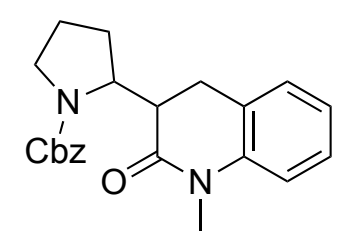

$( \pm)-7 \mathrm{a}$ 


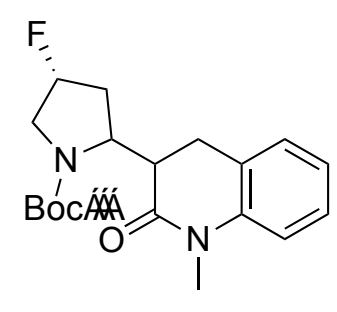

$7 b$

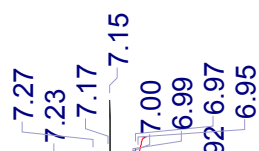
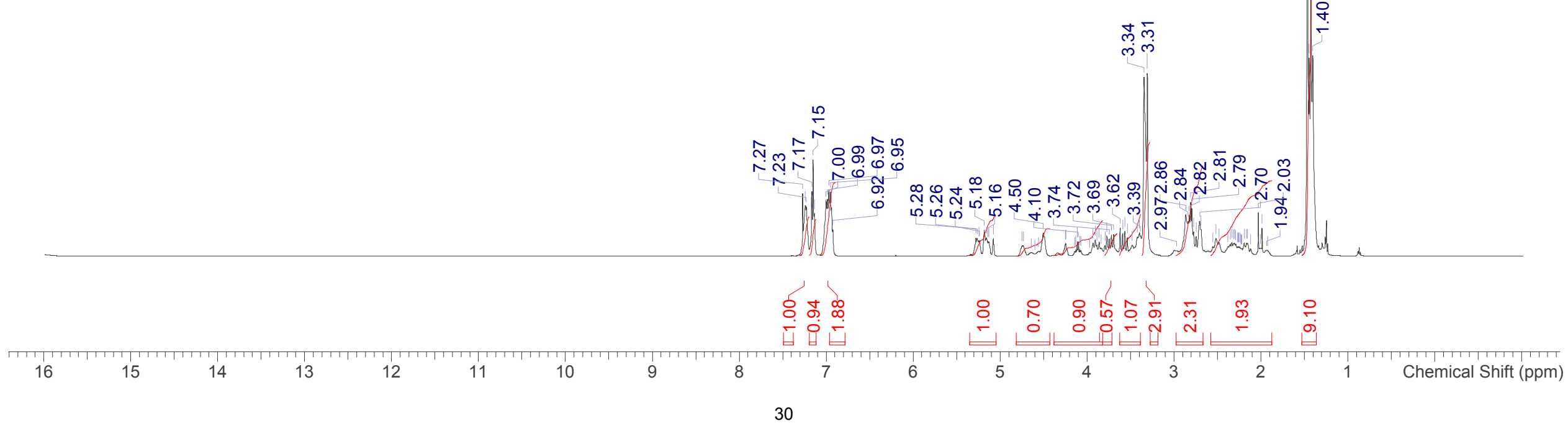


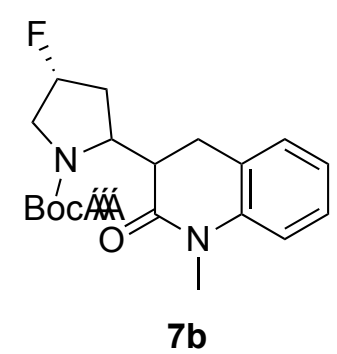

7b

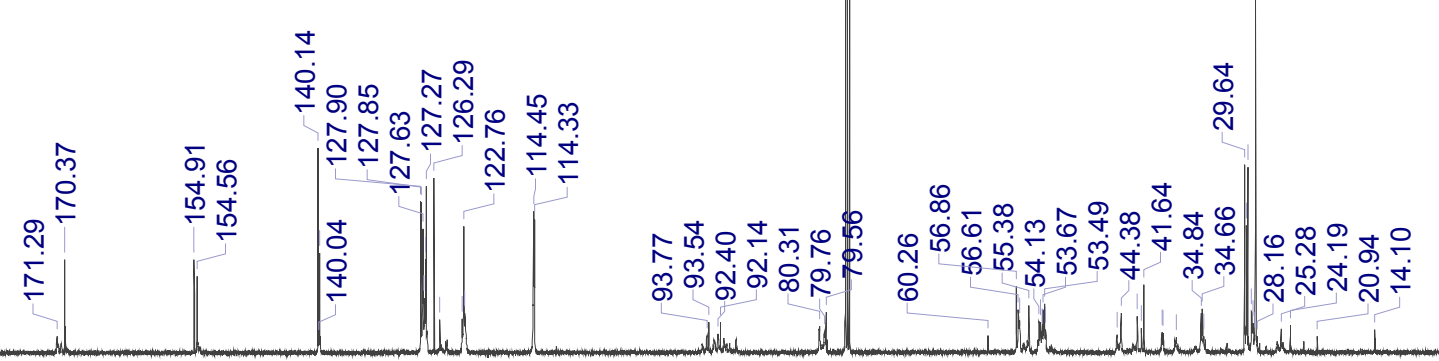




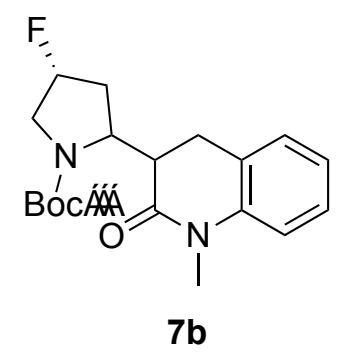

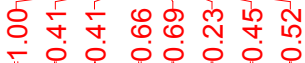




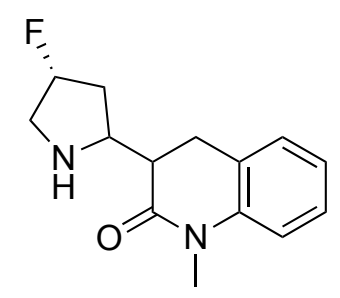

S1 (TFA salt)

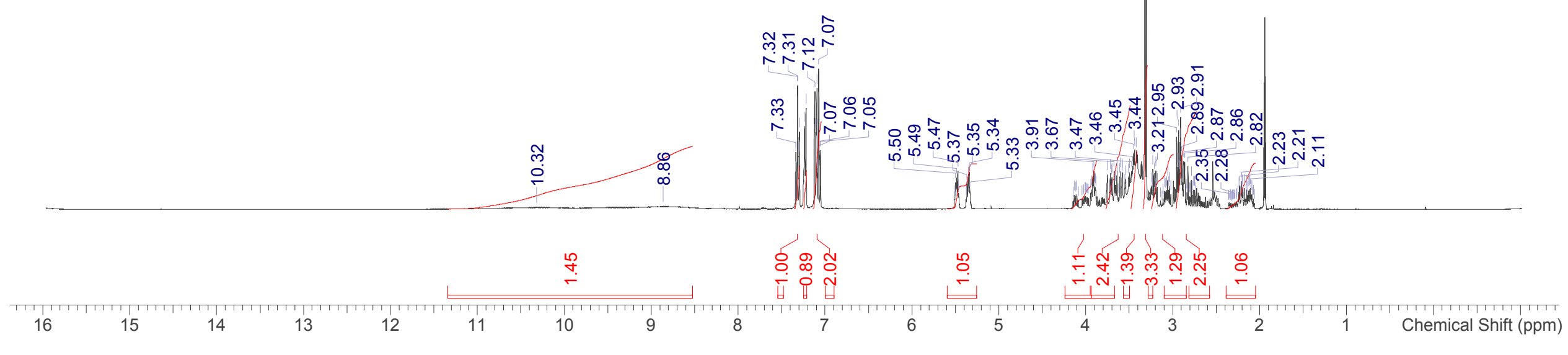




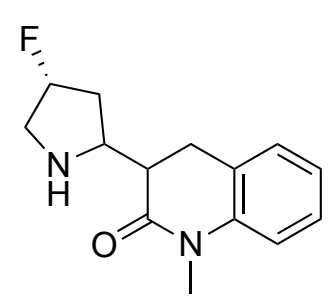

S1 (TFA salt)

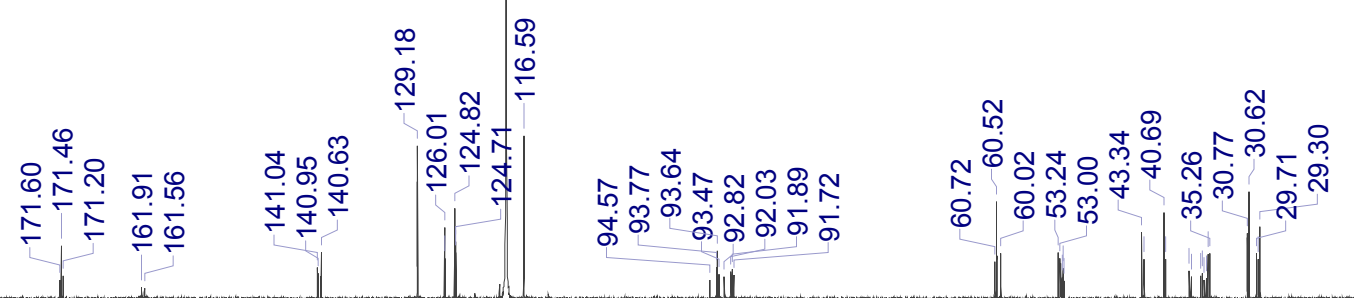




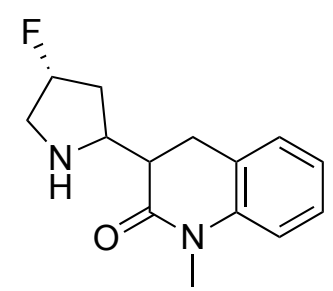

S1 (TFA salt)

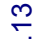

ஓ் ำำ

ำ

$\stackrel{\stackrel{\sim}{N}}{\underset{N}{N}}$

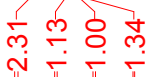

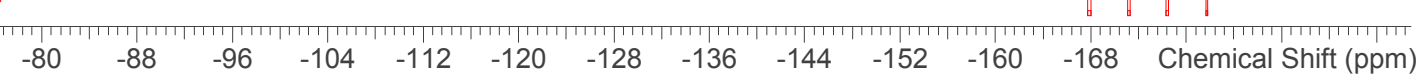




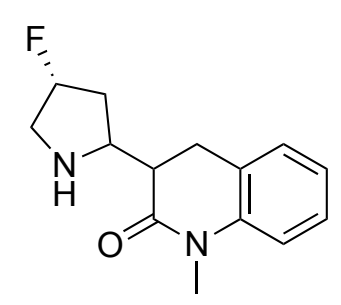

S1 (TFA salt)
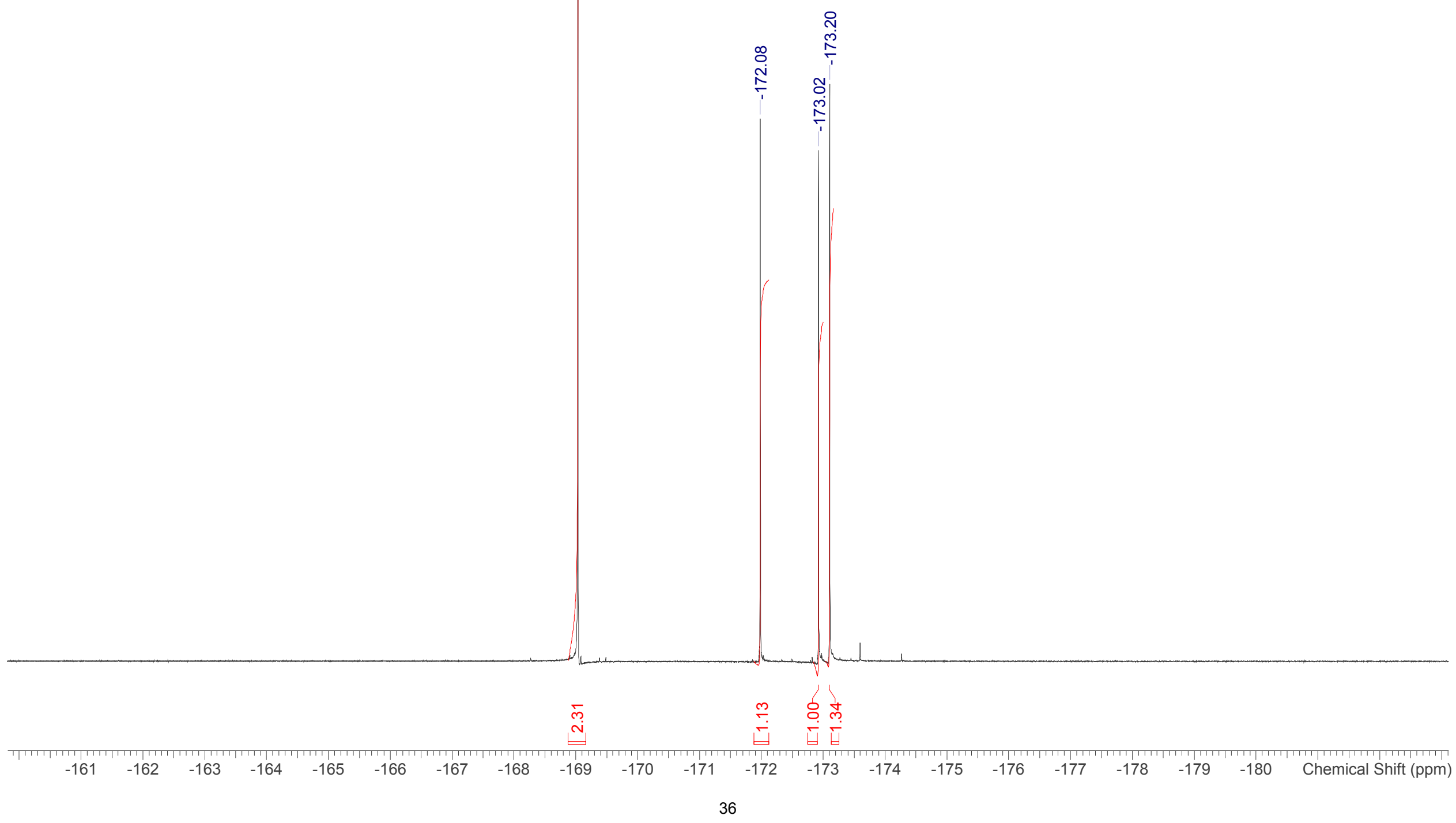


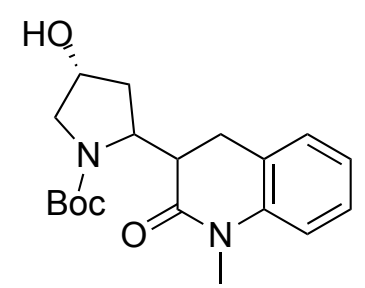

$( \pm)-7 c$

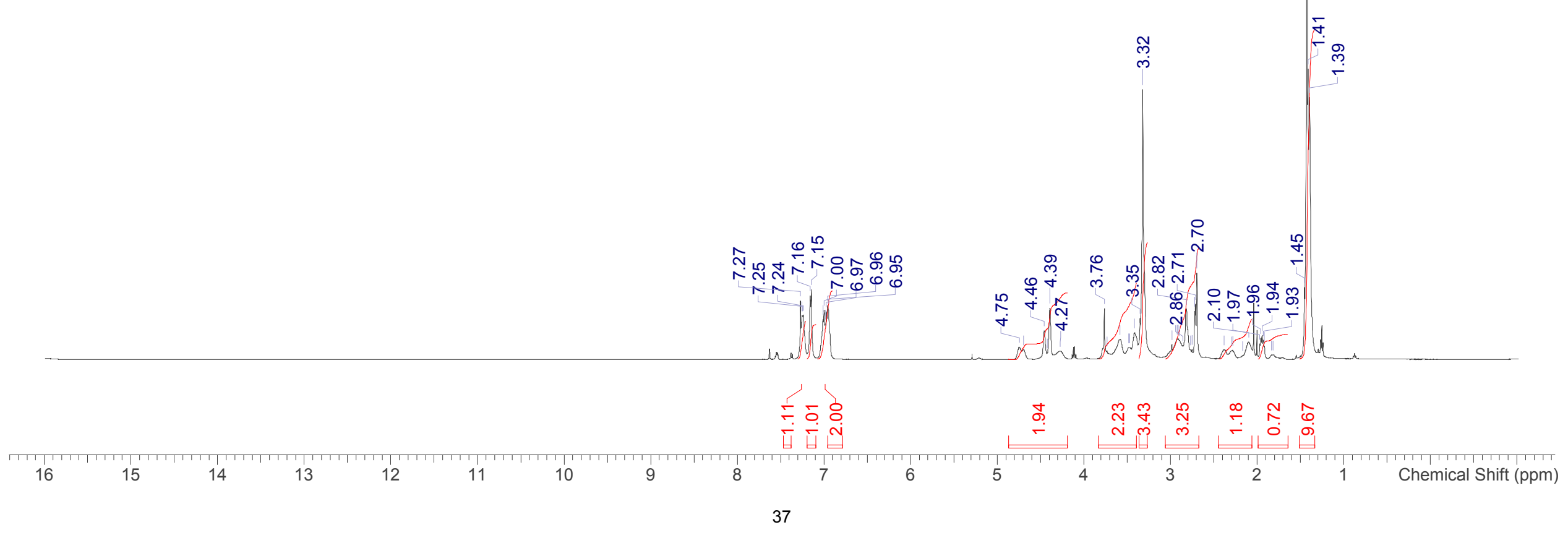




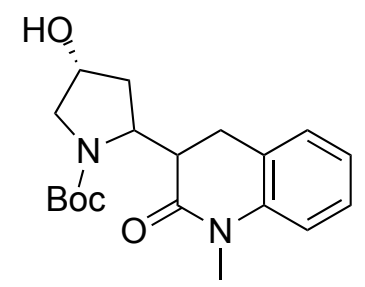

$( \pm)-7 \mathrm{c}$

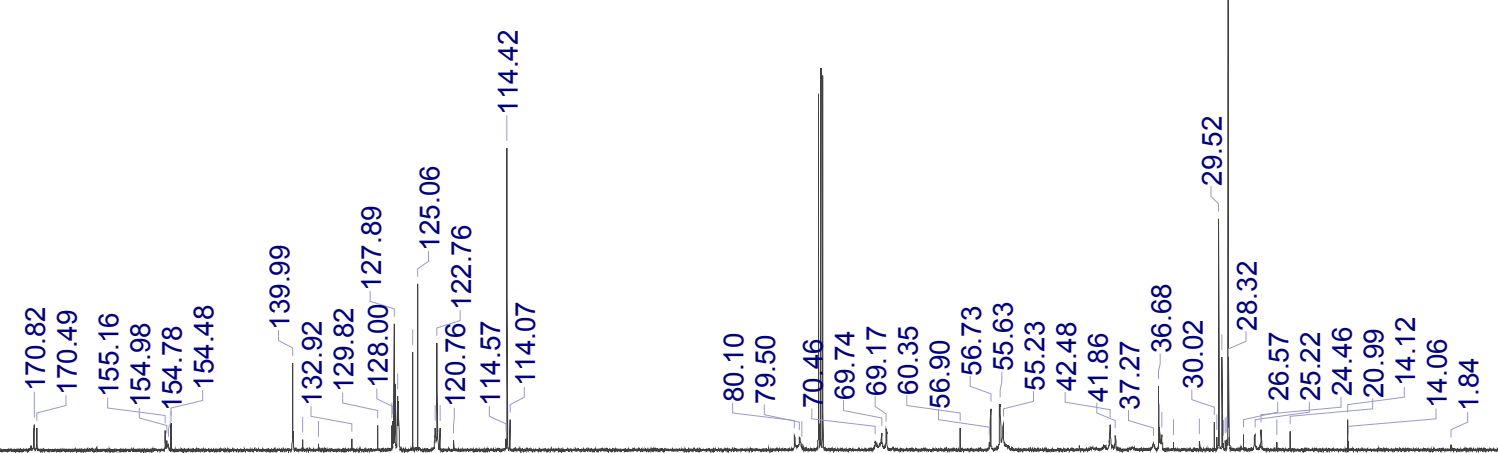




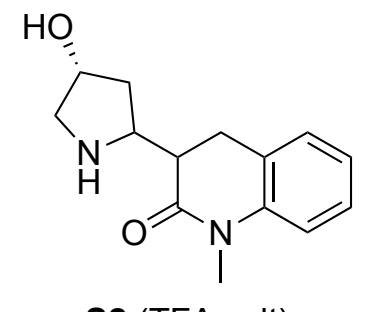

S2 (TFA salt)

స్

$\stackrel{\infty}{\infty}$

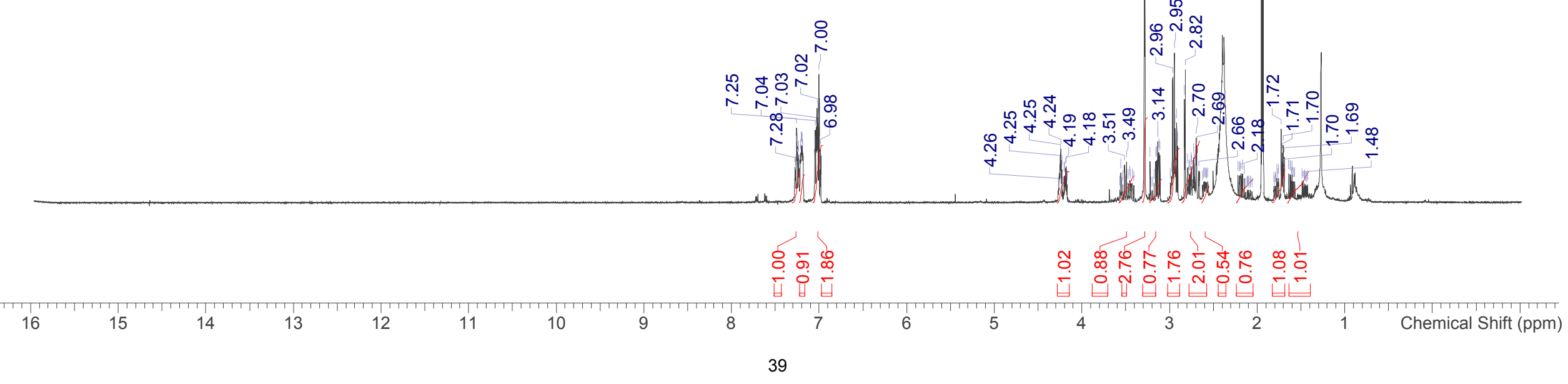

욜 용 


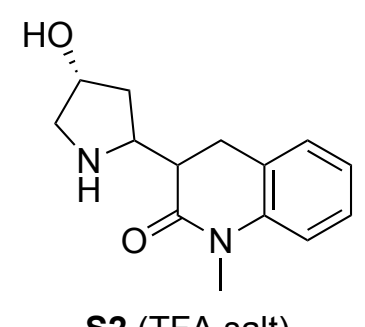

S2 (TFA salt)

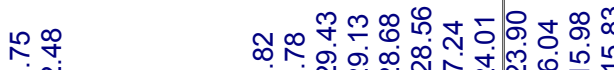

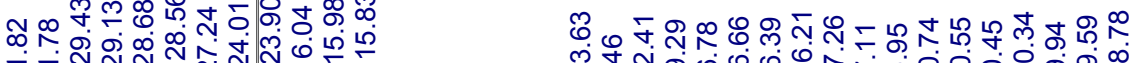

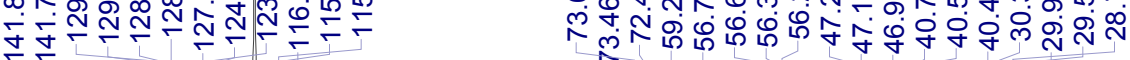



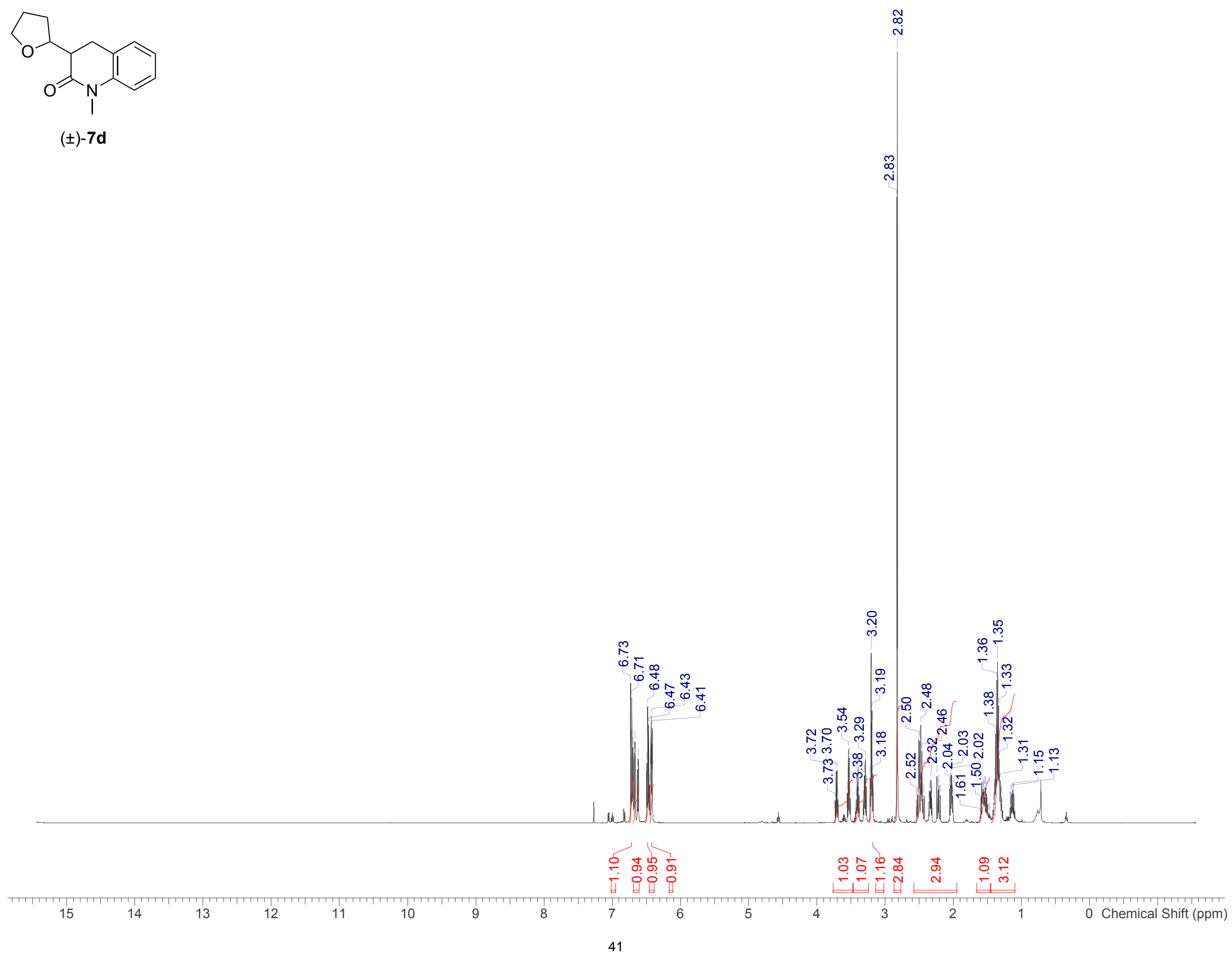


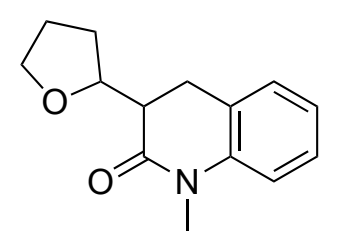

( \pm -7d

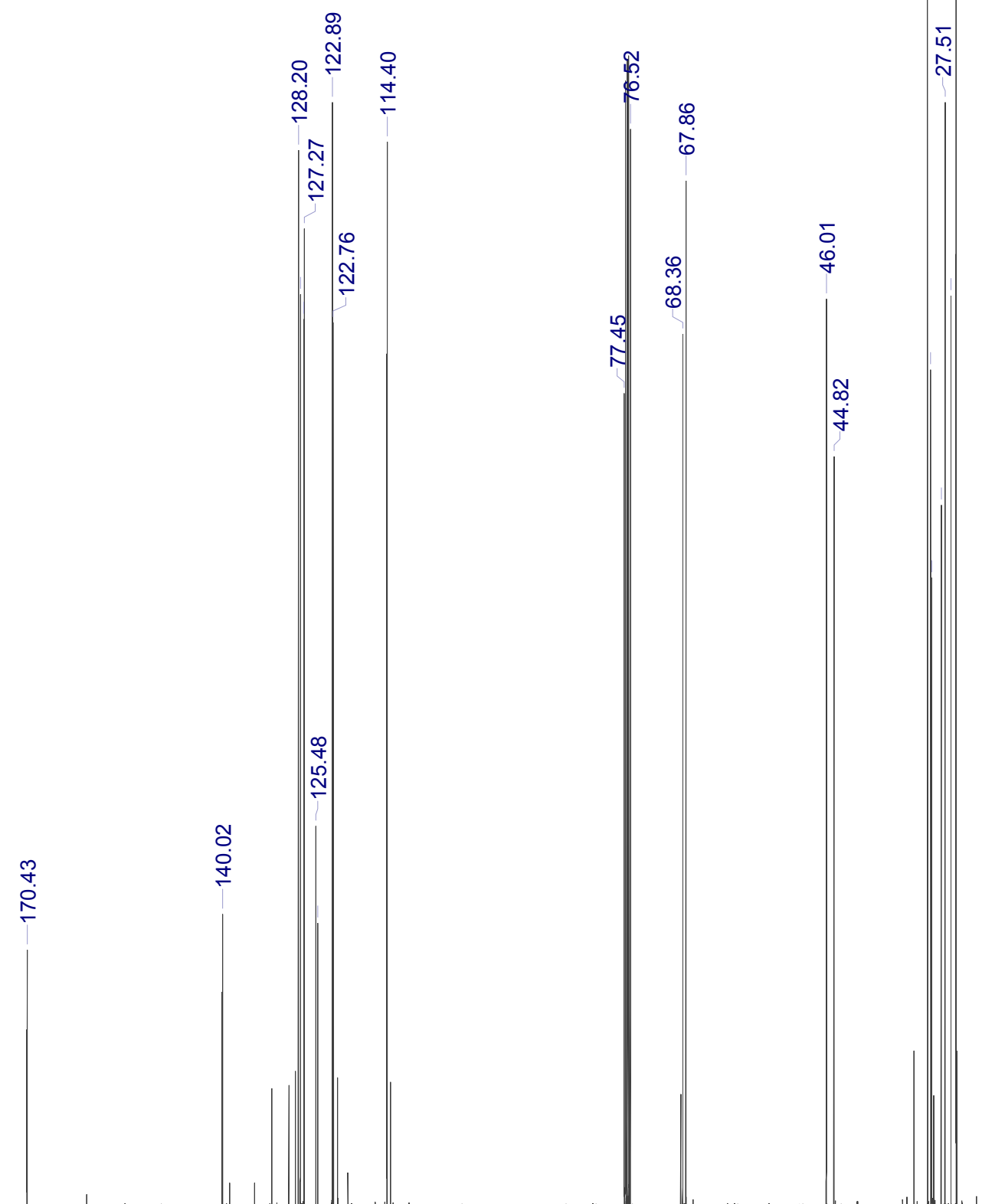

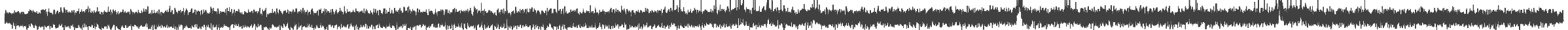

$\uparrow \quad \infty$ 


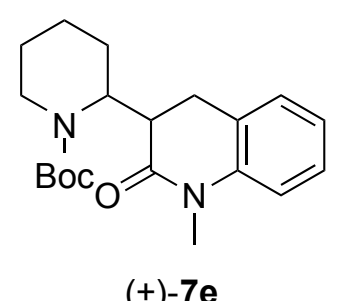

$( \pm)-7 e$

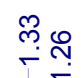

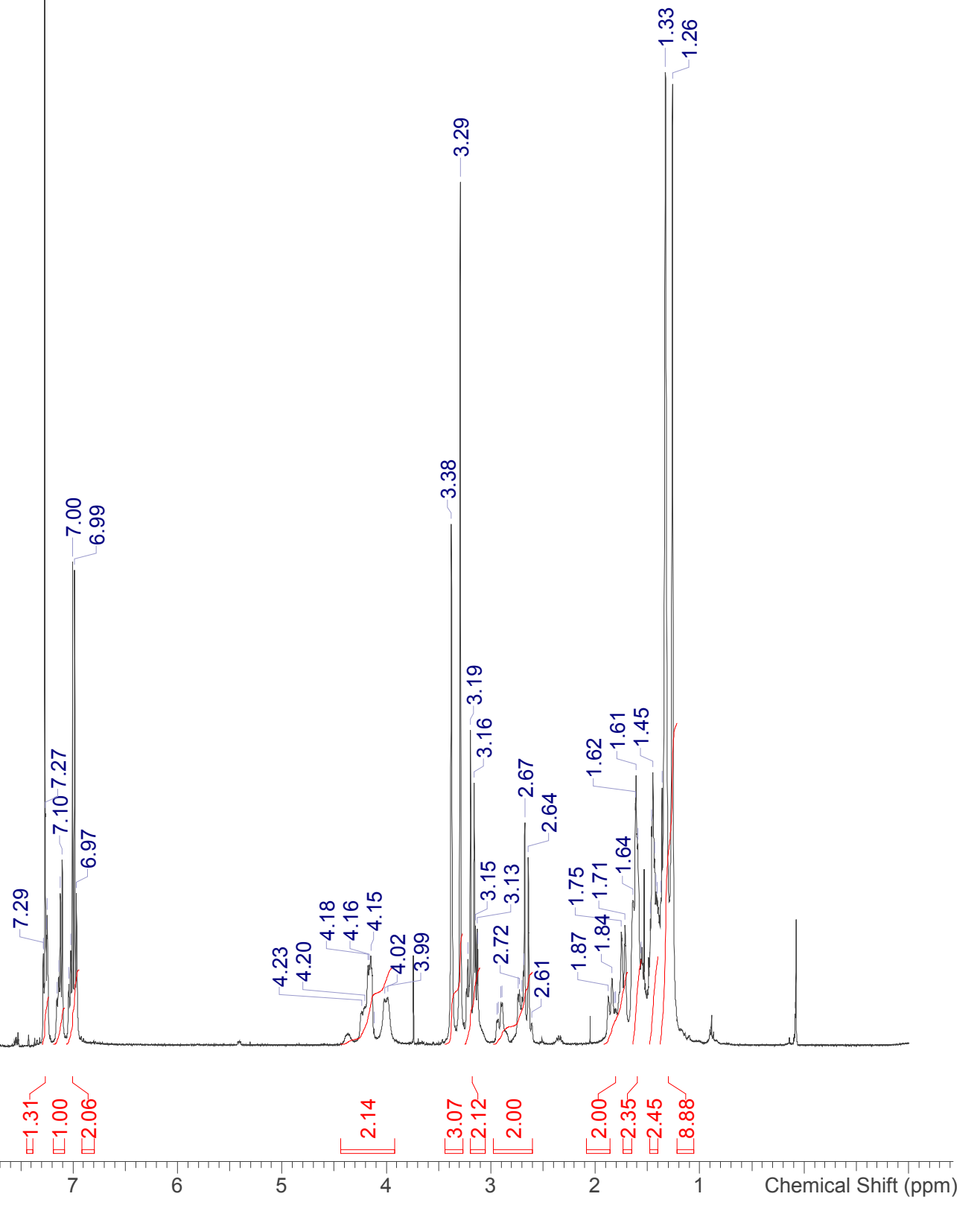



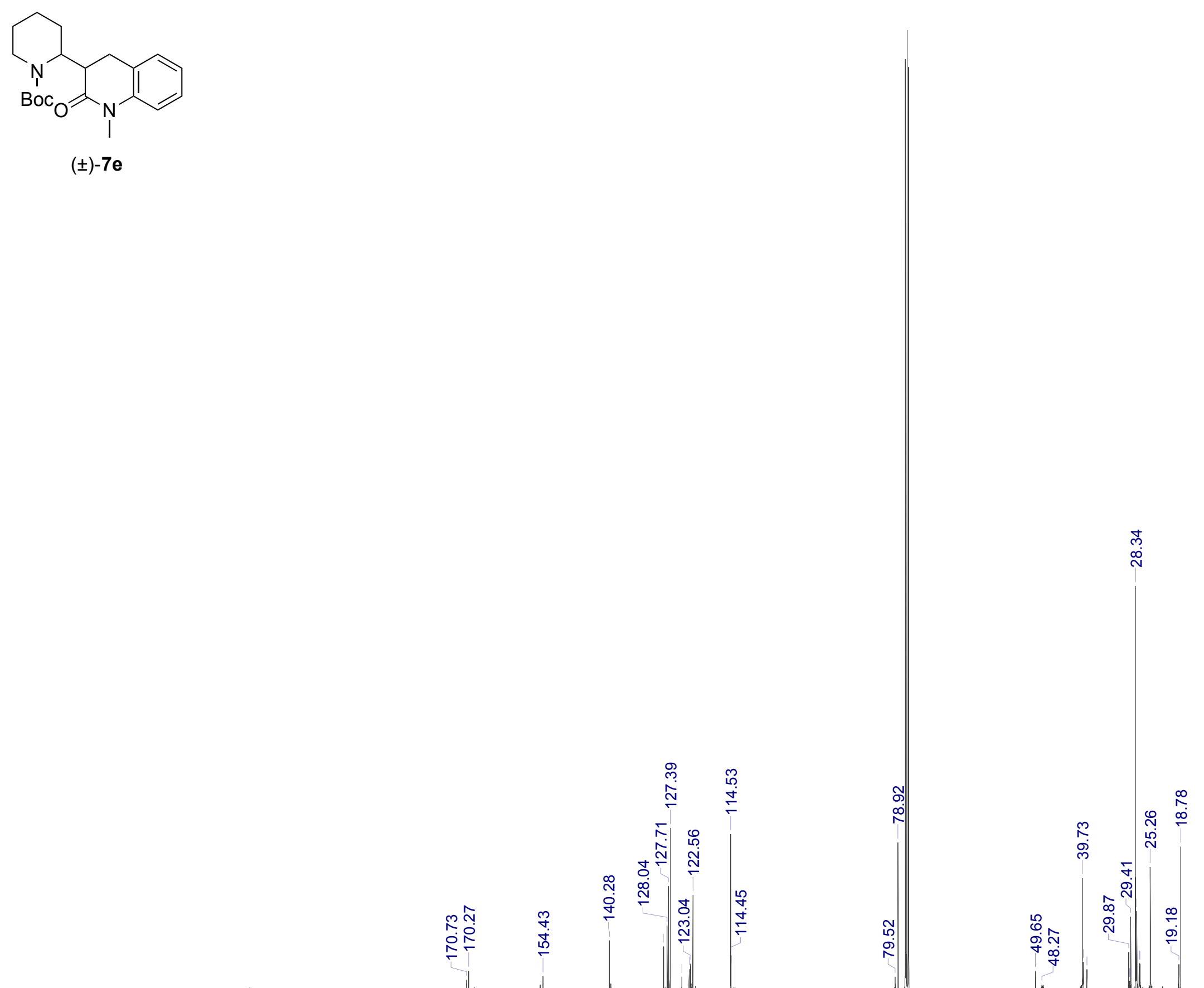


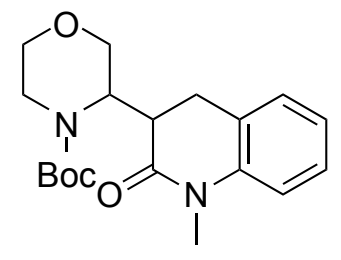

$( \pm)-7 f$

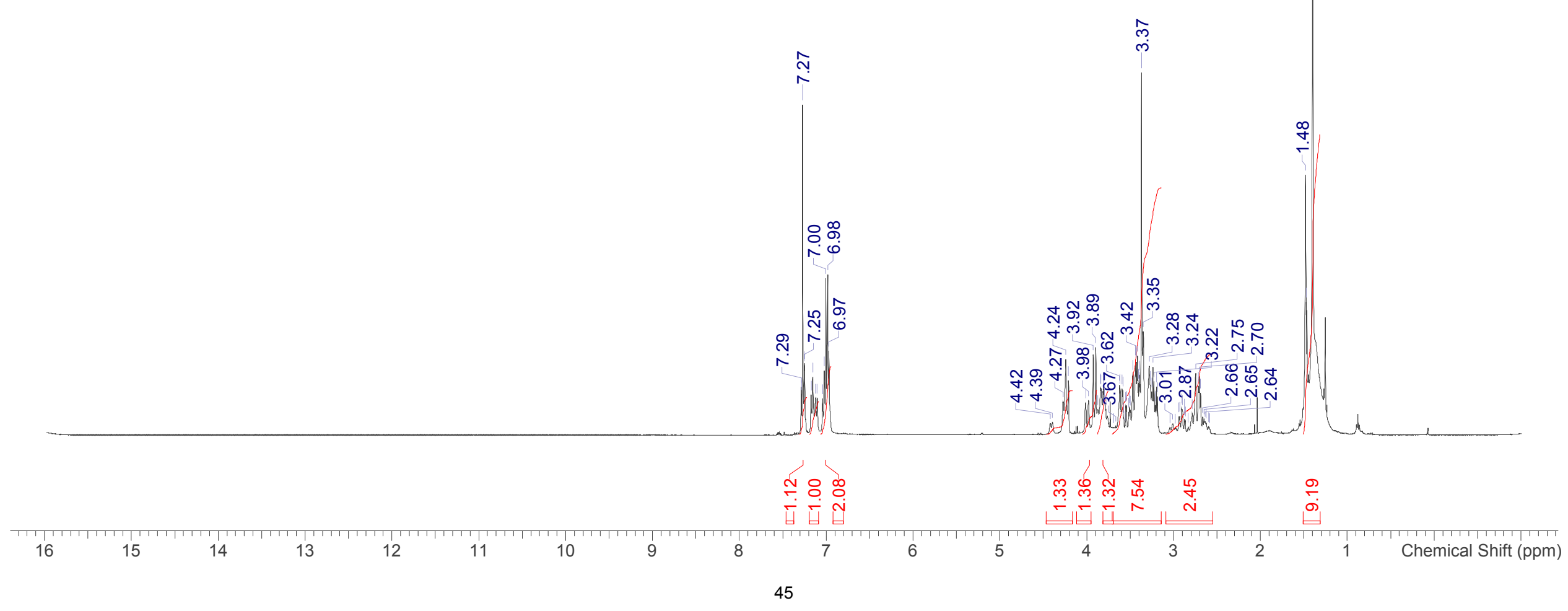




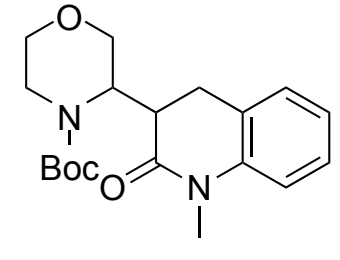

$( \pm)-7 f$

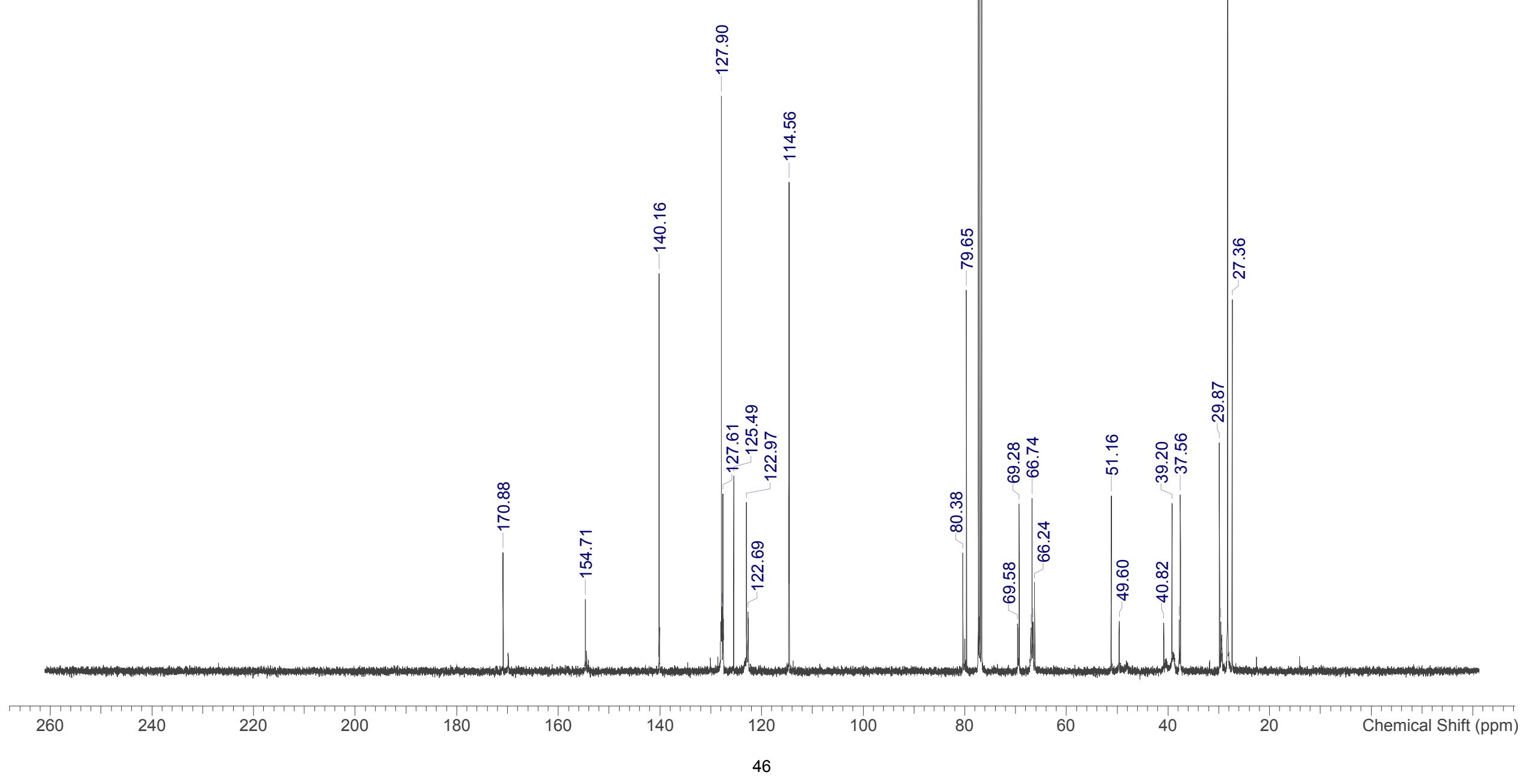




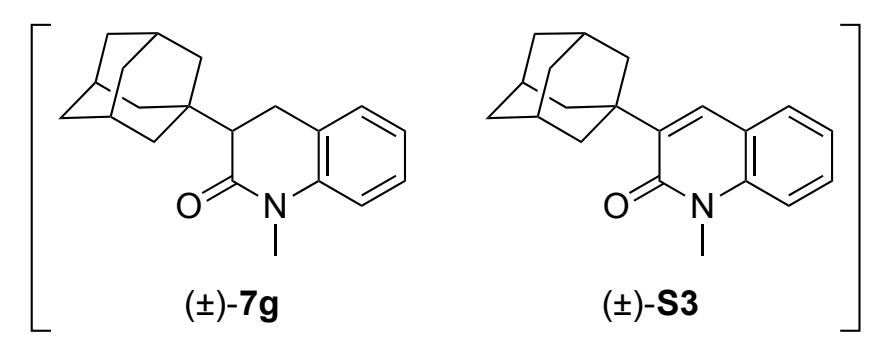

$\underset{i}{i}$
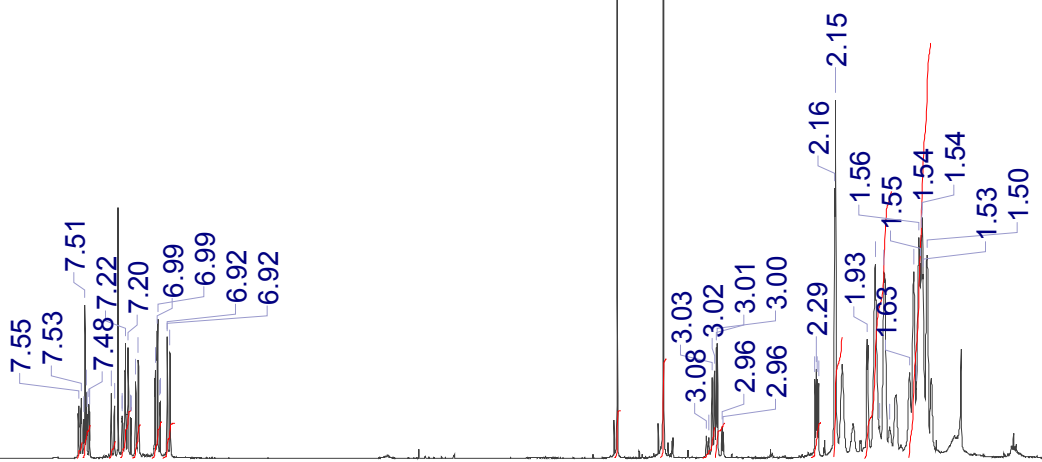

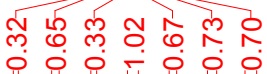

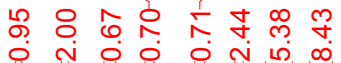




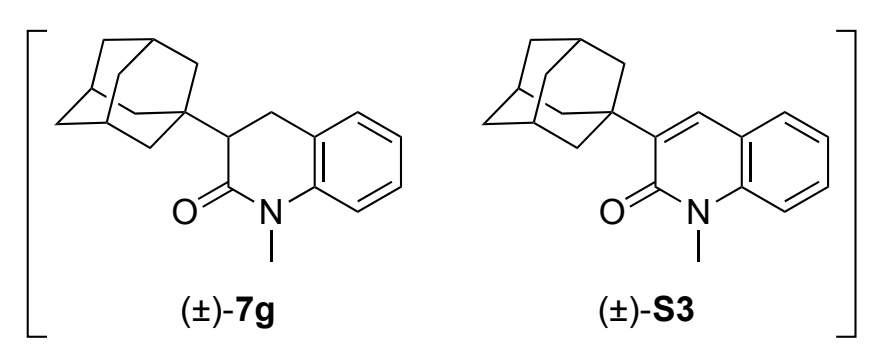

$\substack{0 \\ \infty \\ \infty}$
N

菅

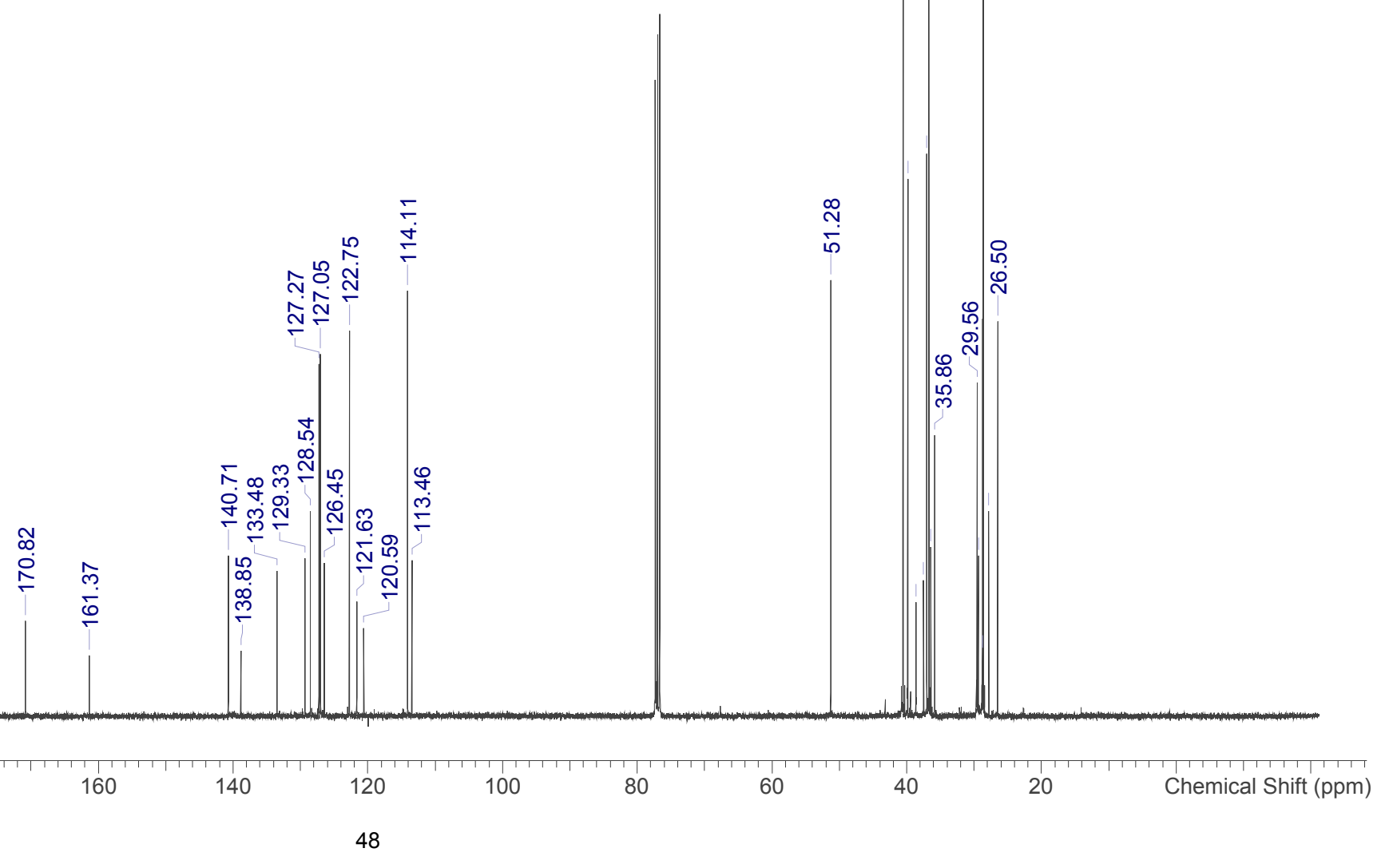




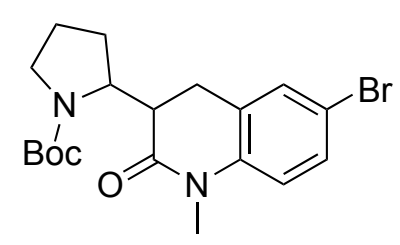

( \pm -9a

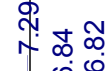

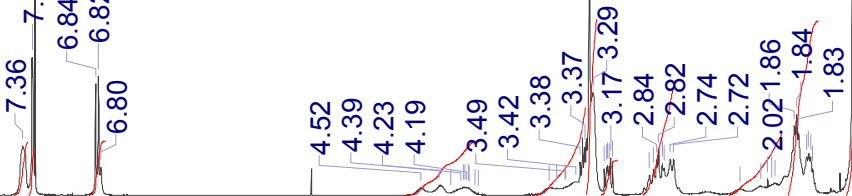

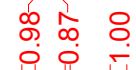
¿. 


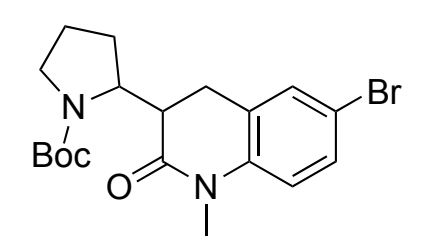

$( \pm)-9 a$

ㅎํ

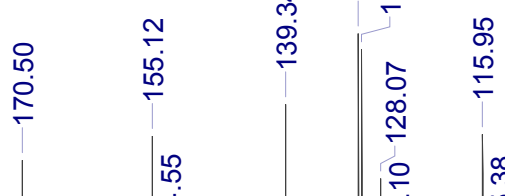

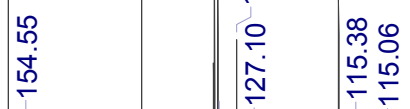

\&

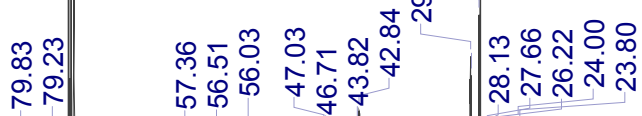




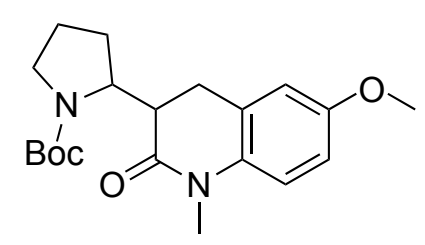

( \pm -9b

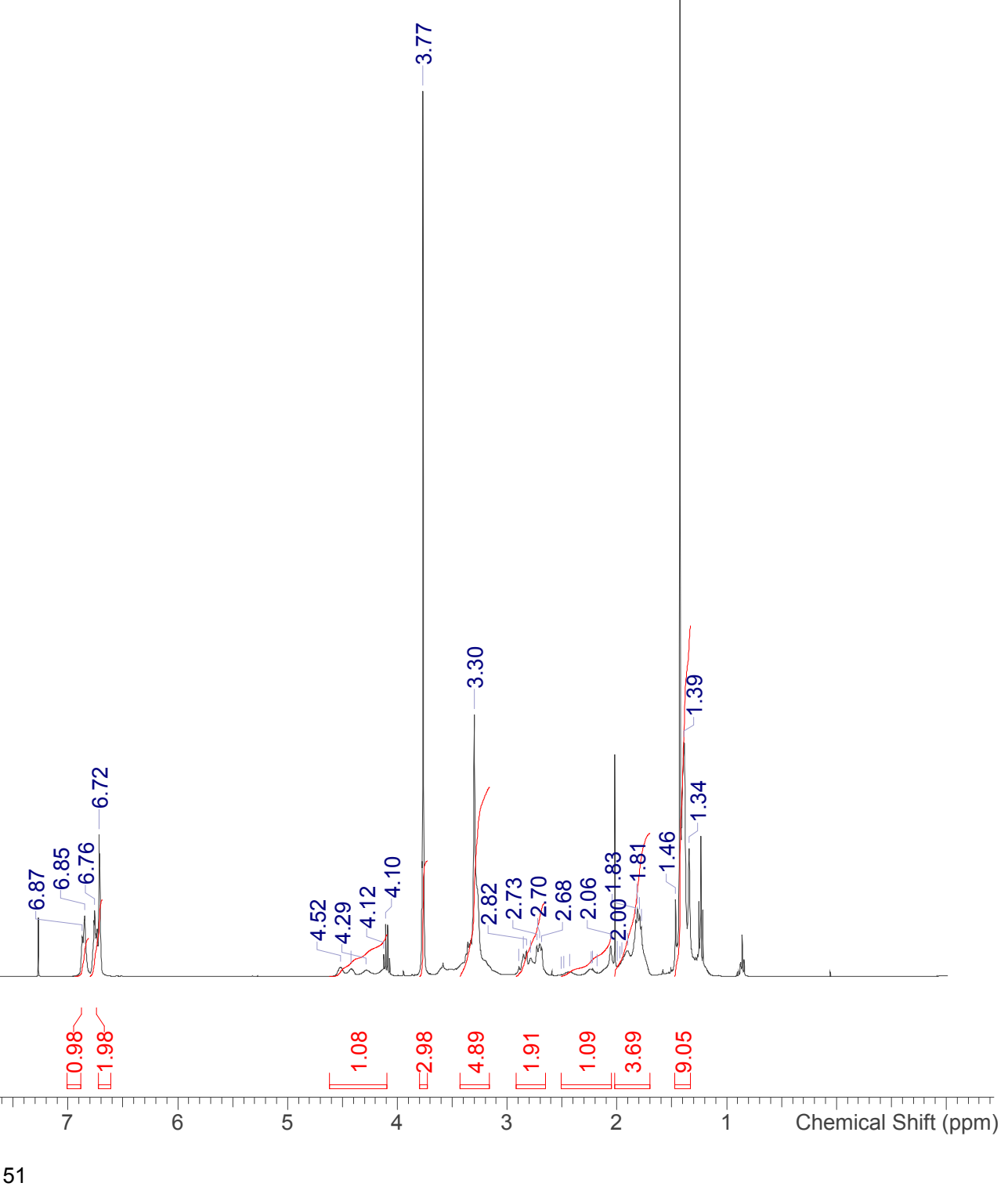




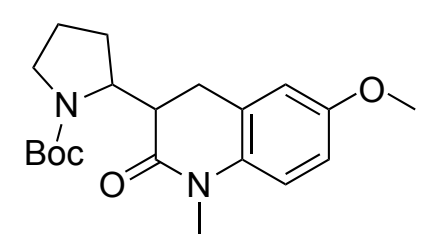

( \pm - $-9 b$

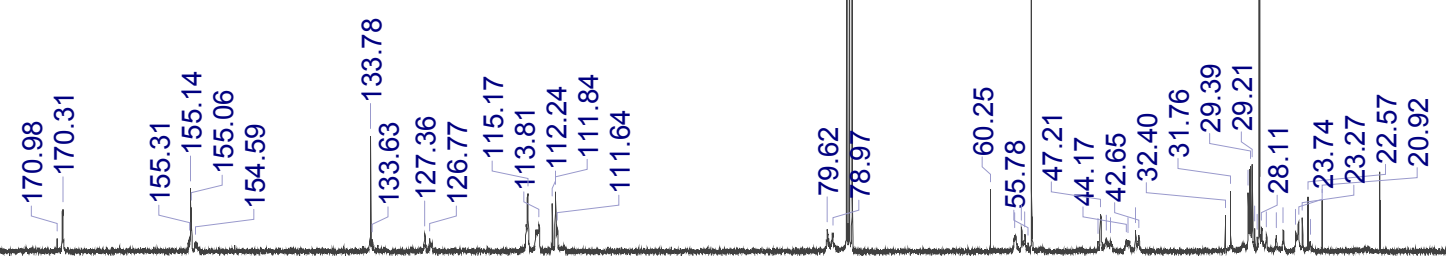




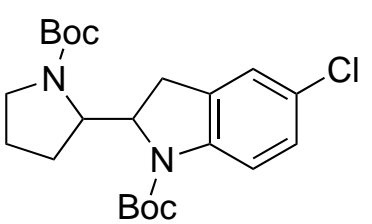

$( \pm)-9 c$

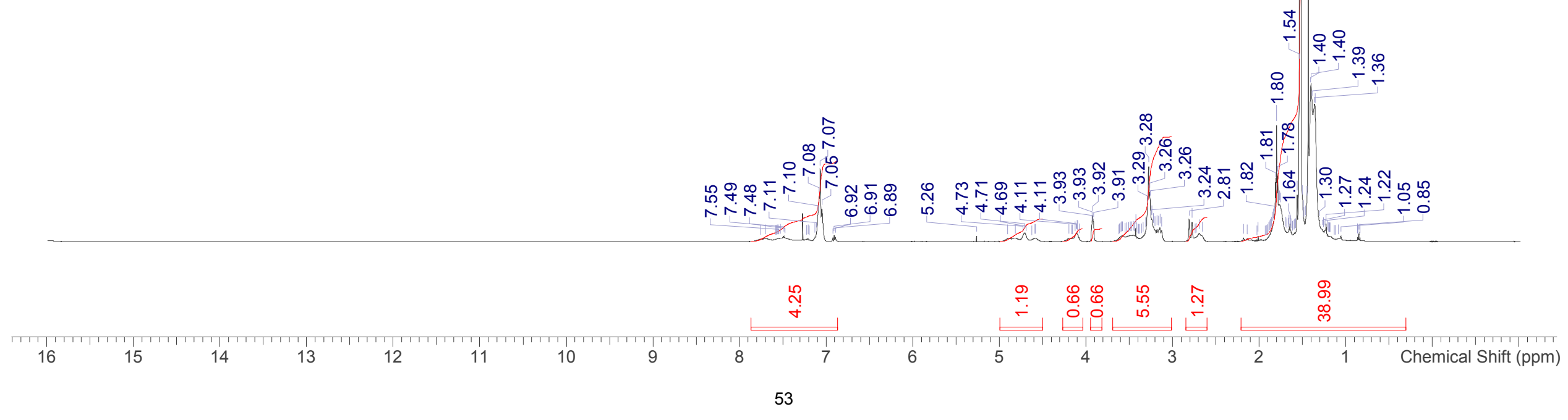




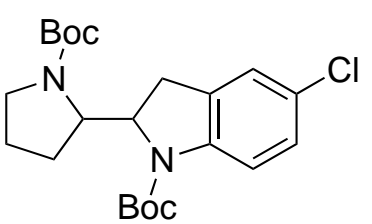

( \pm -9c 


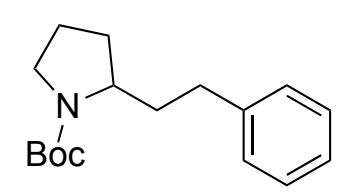

( \pm -9d

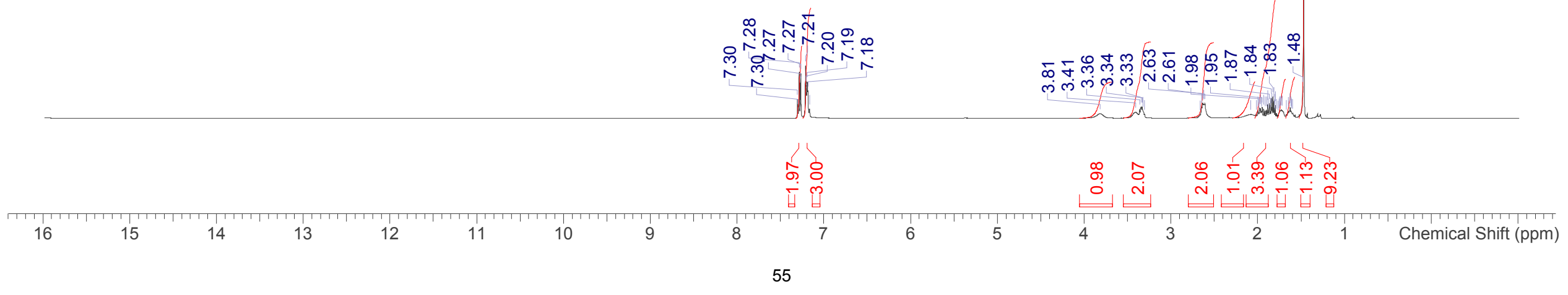




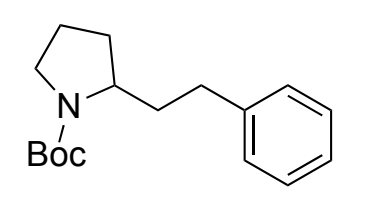

( \pm -9d

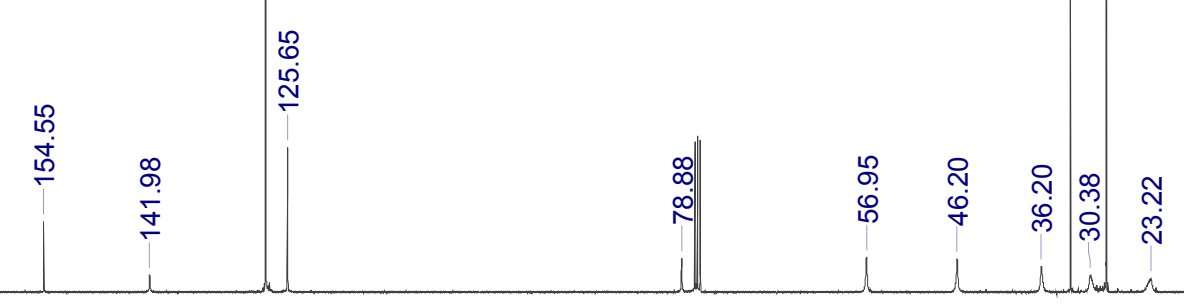




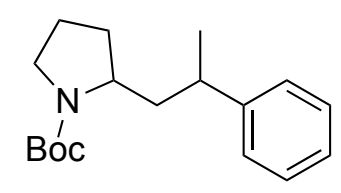

$( \pm)-9 e$

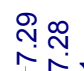

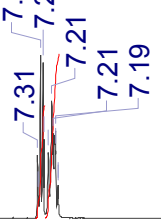

œ

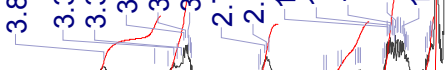

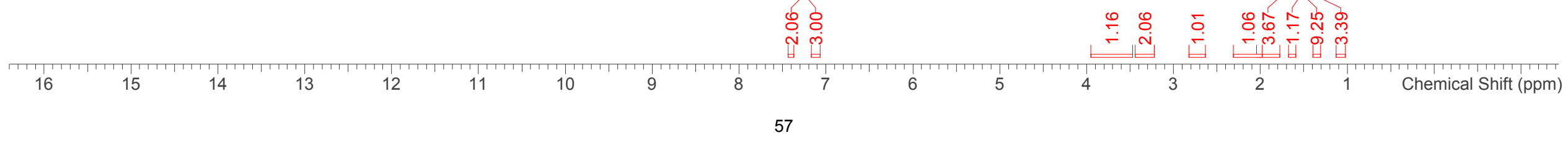




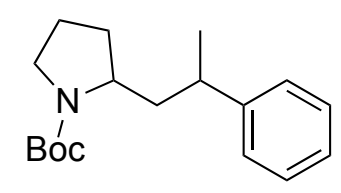

$( \pm)-9 e$

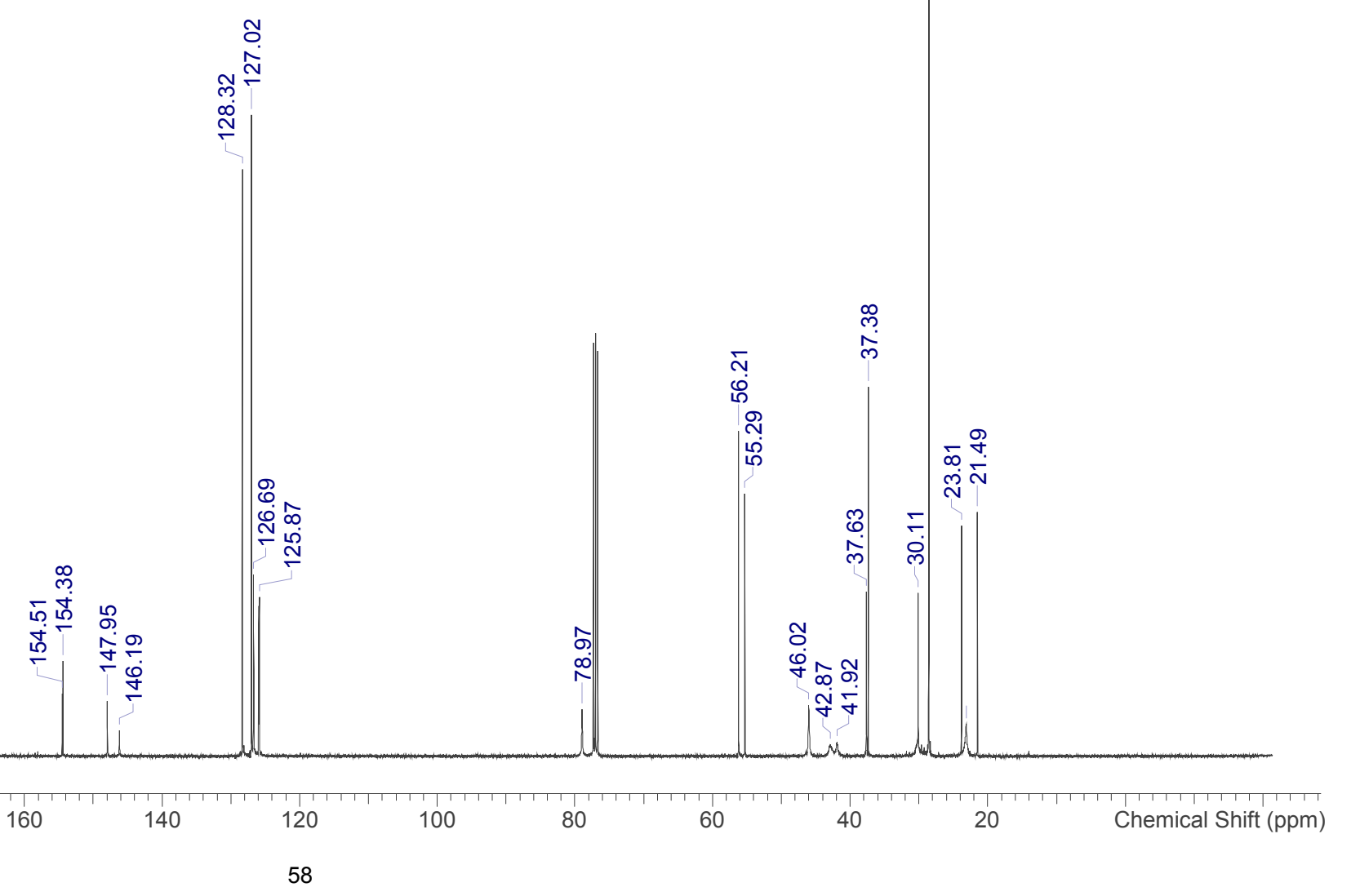




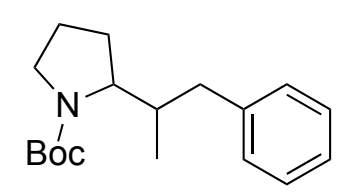

( \pm -9f

$\stackrel{\text { กิ }}{\wedge}$

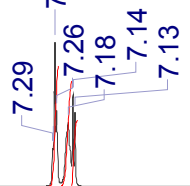

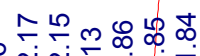

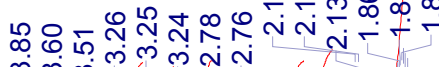

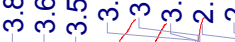

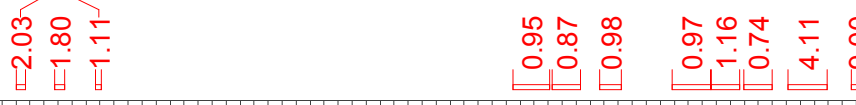




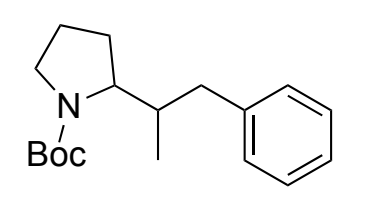

$( \pm)-9 f$

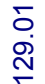

$\stackrel{ }{\stackrel{\infty}{\infty}}$

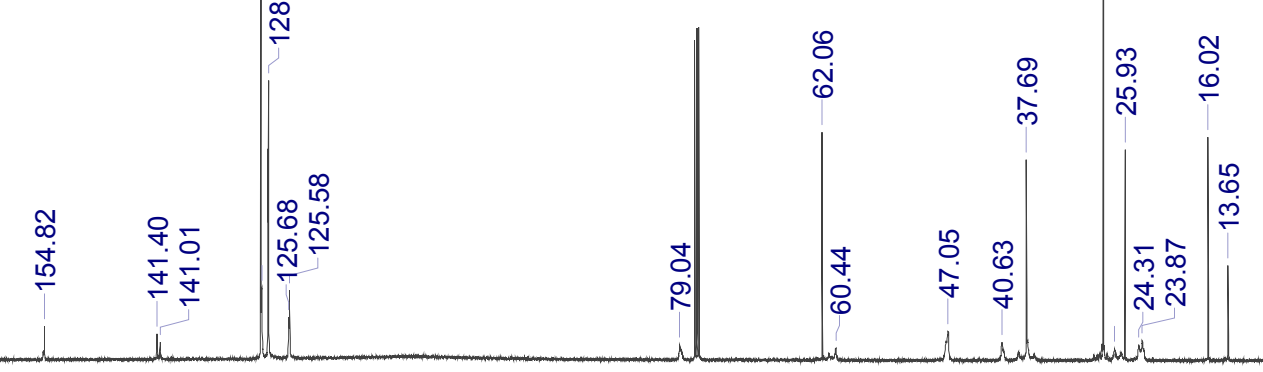




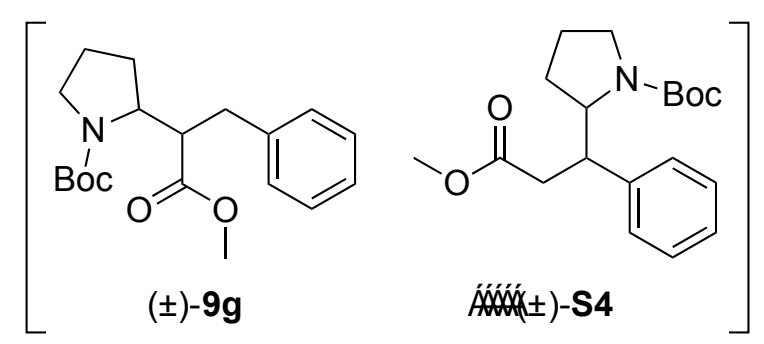

in

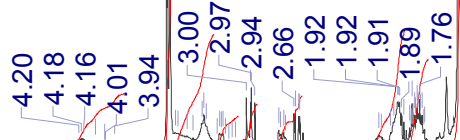

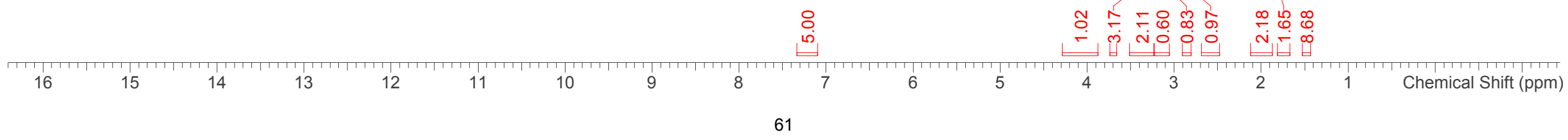



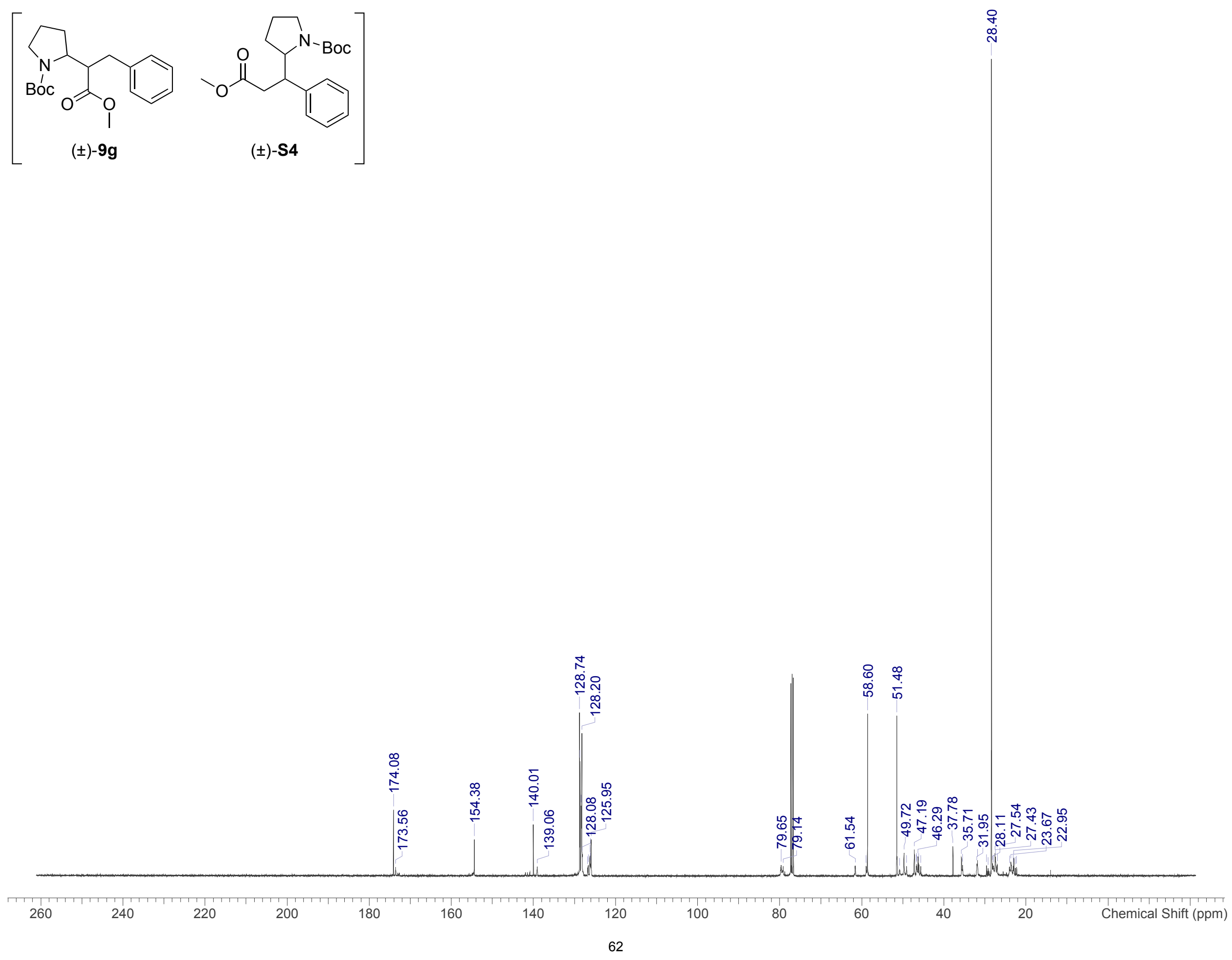

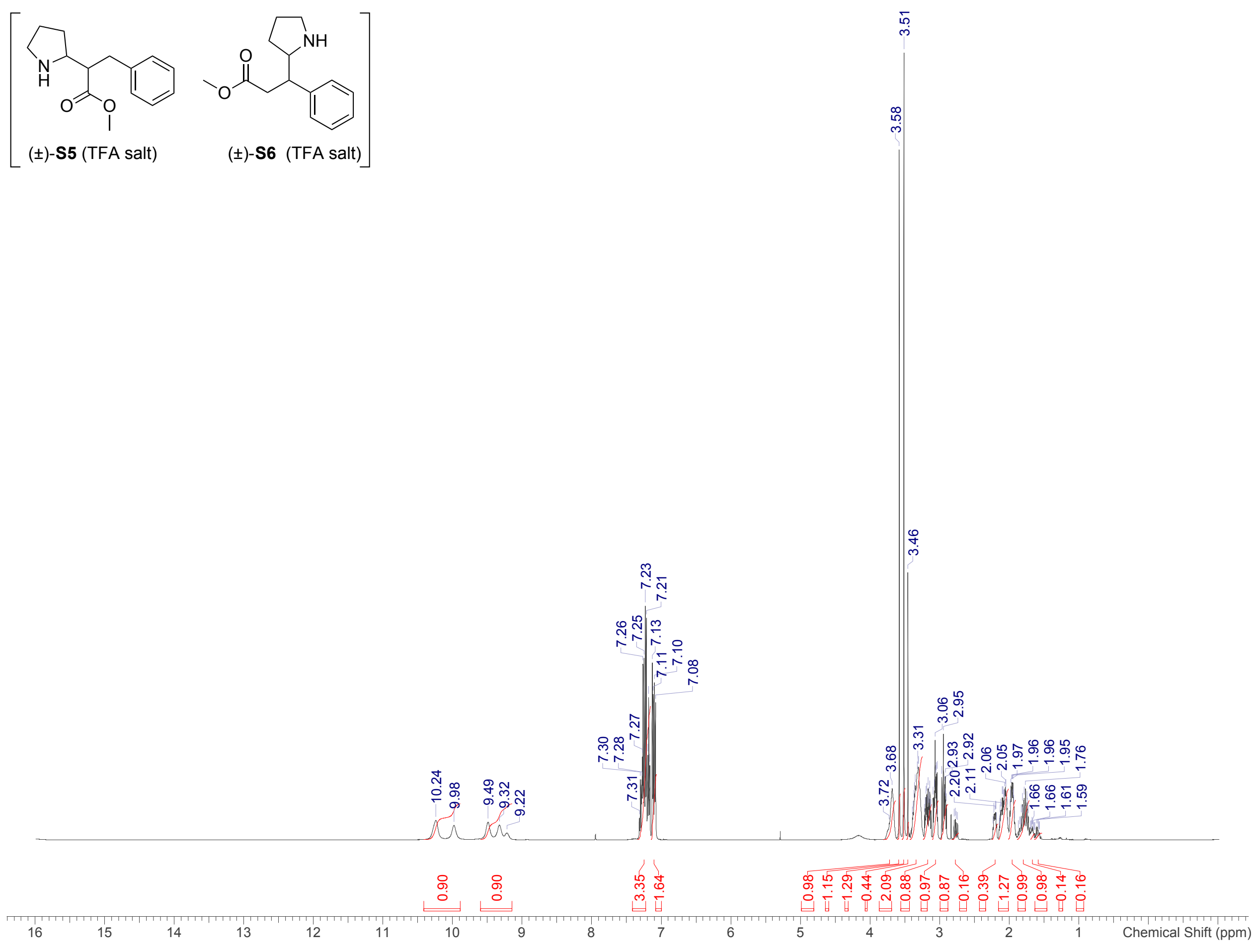

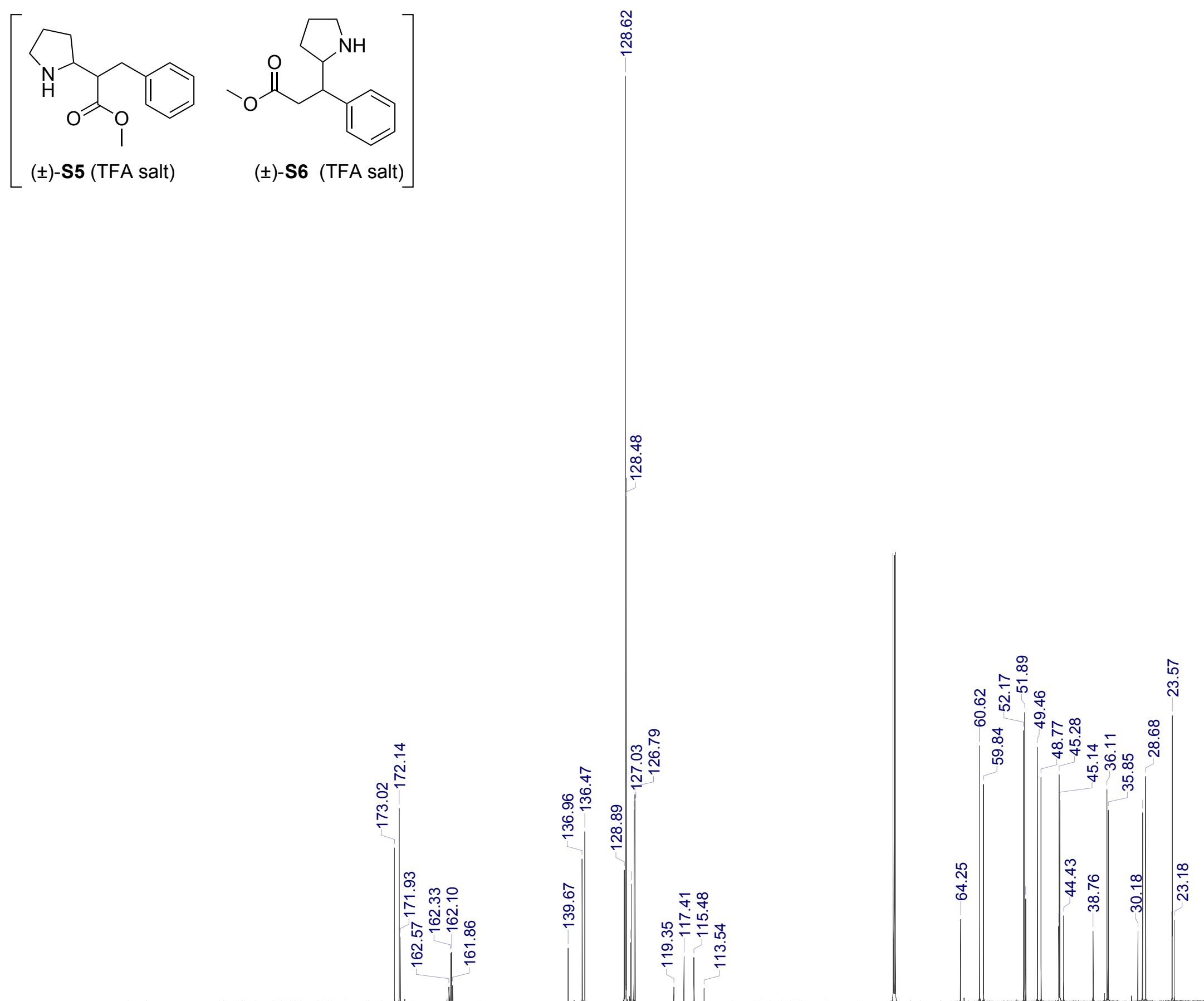


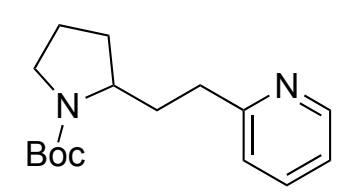

( $)-9 h$

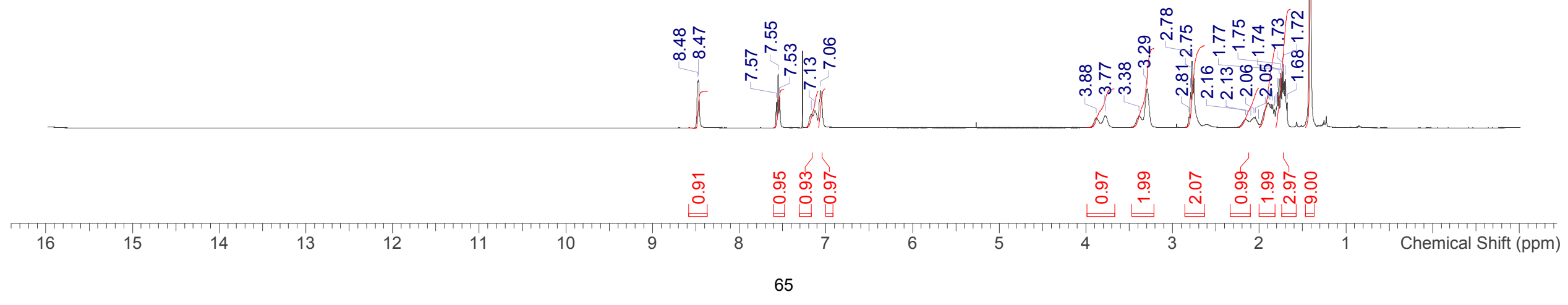




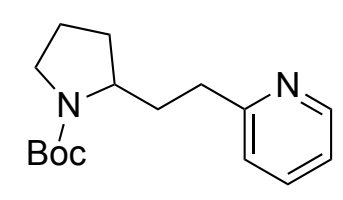

$( \pm)-9 h$

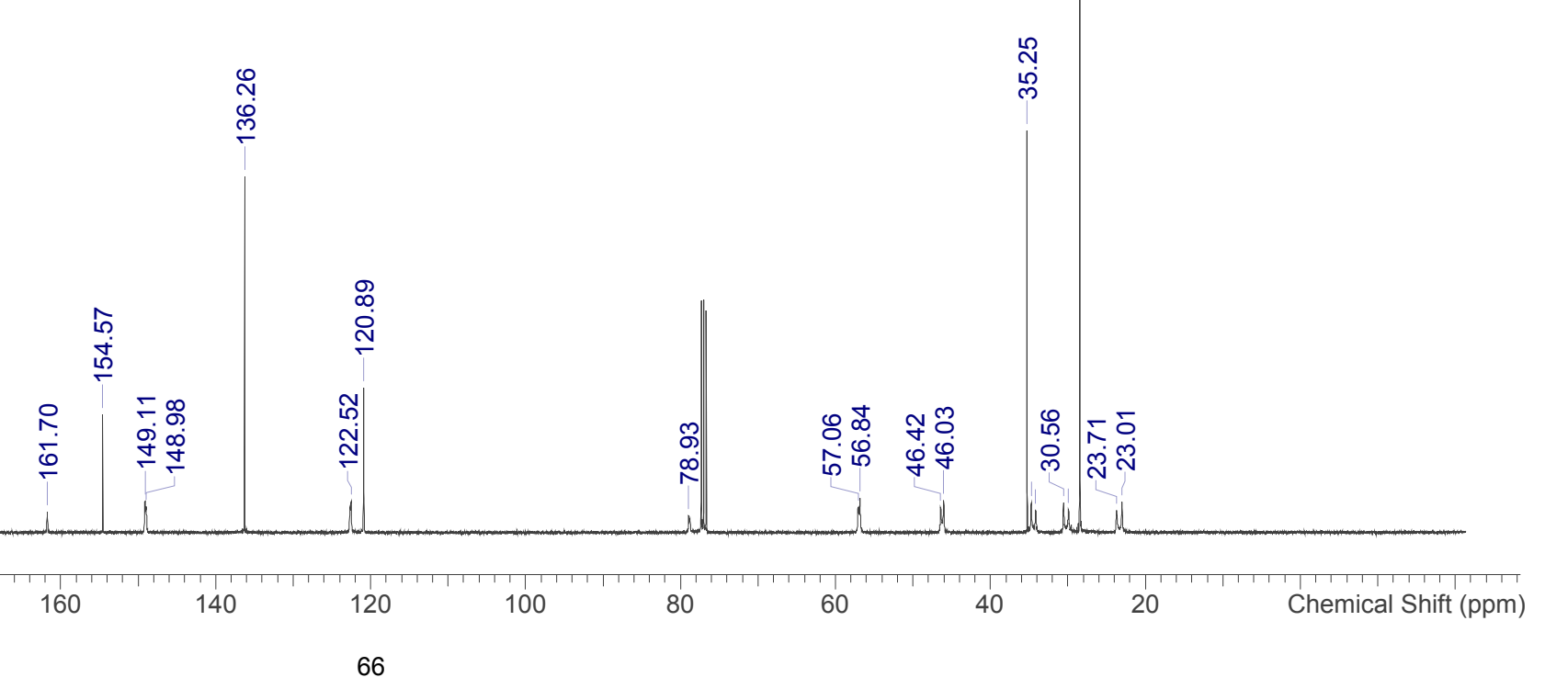




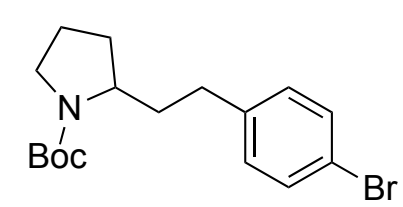

$( \pm)-9 \mathbf{i}$

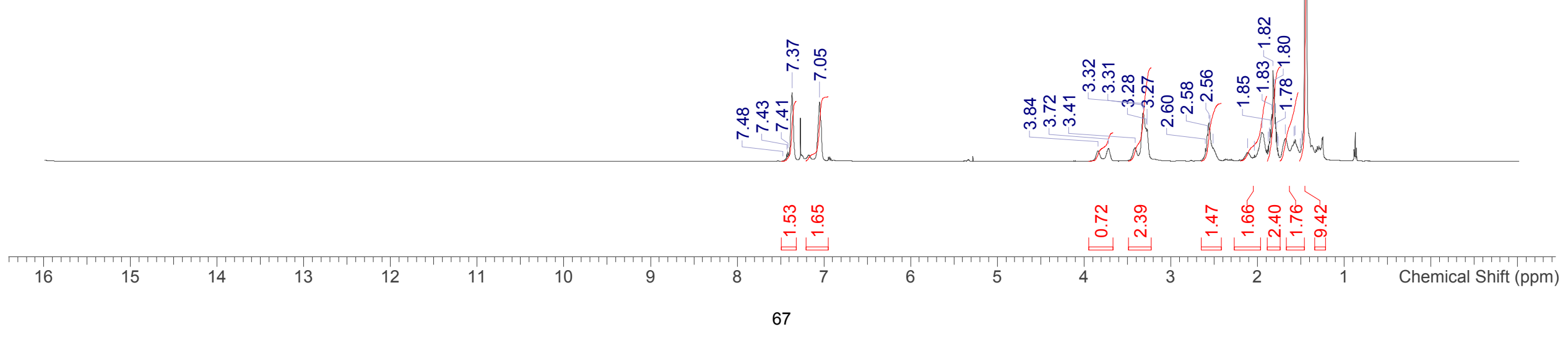




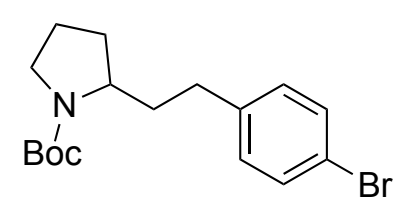

( $)-9 \mathbf{i}$

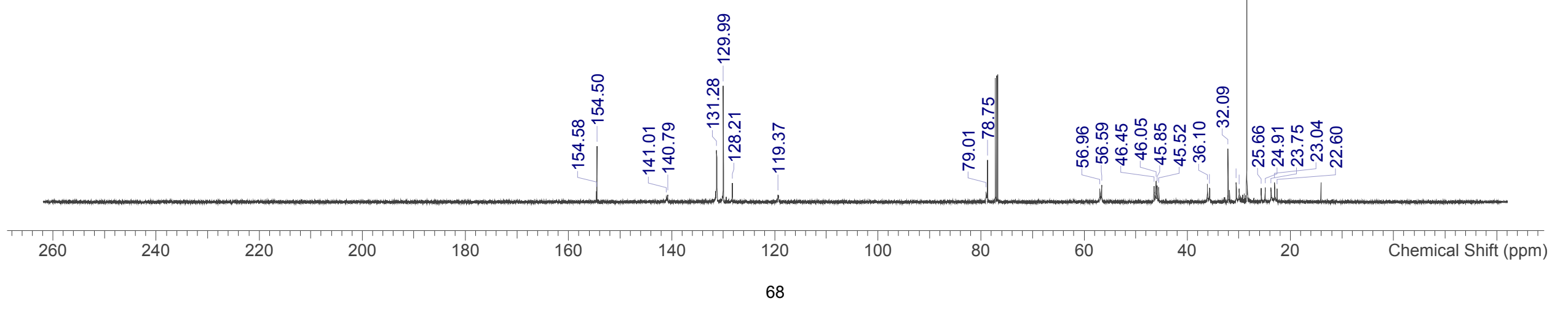



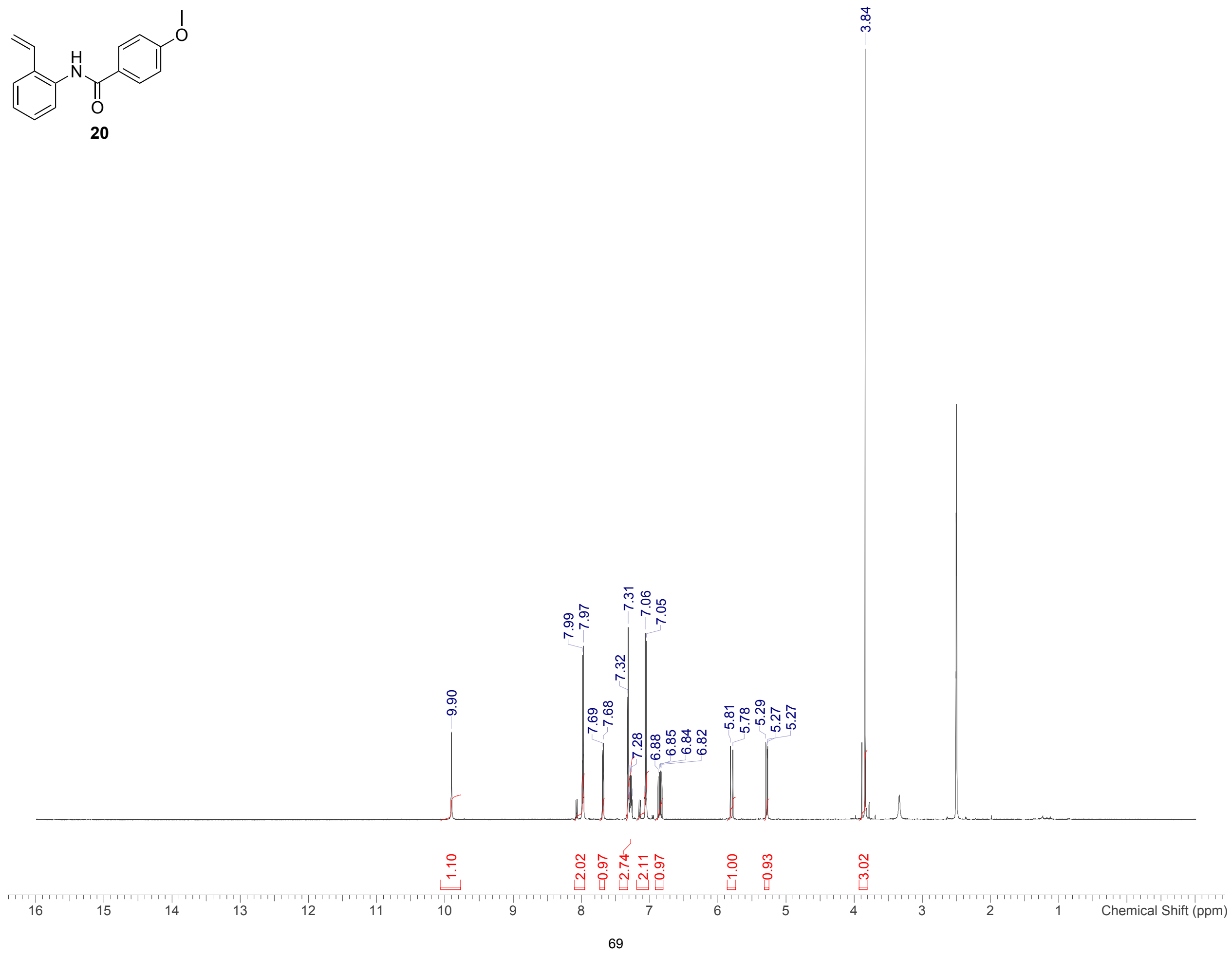


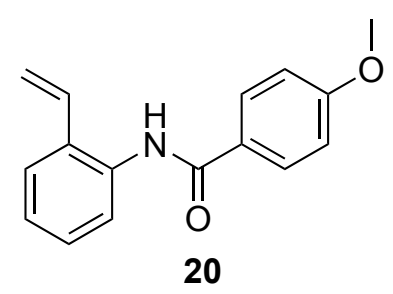

๙ั)

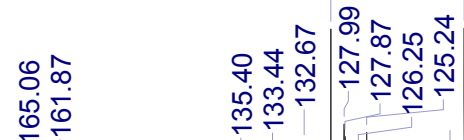

弄 


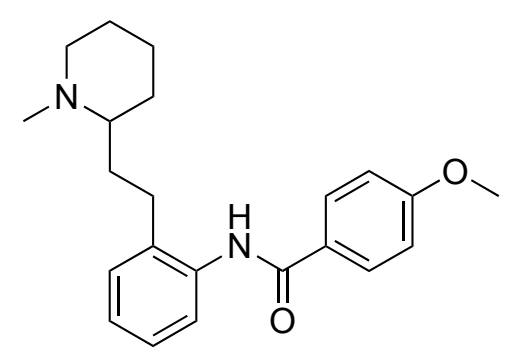

( \pm -17 (encainide)
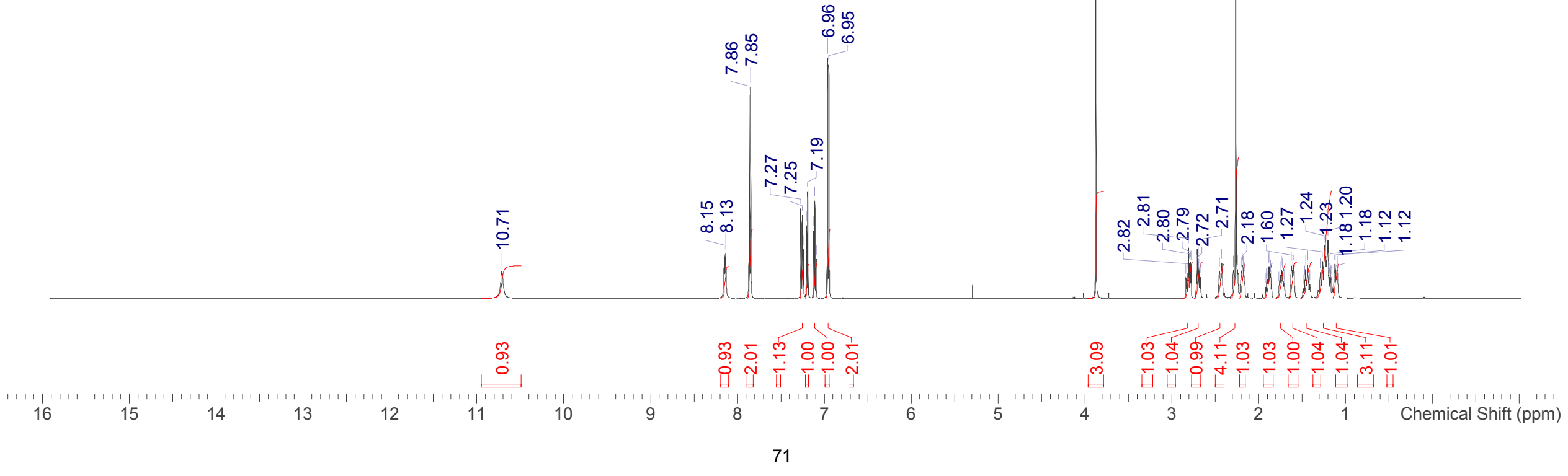


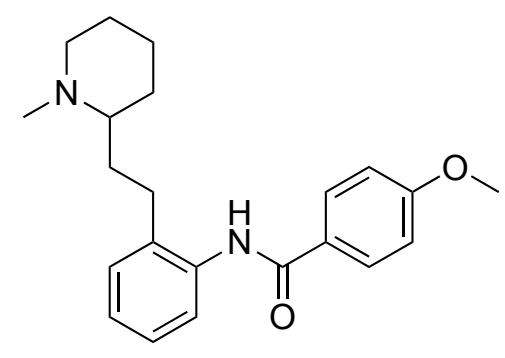

( \pm -17 (encainide)

원

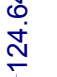

@.

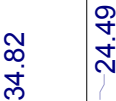

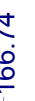

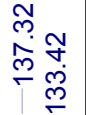

